Key Words:

Aluminosilicate

Neutralization

Uranium

Retention:

Permanent

RESULTS OF THE 2H EVAPORATOR ACID CLEANING AND IN-POT NEUTRALIZATION

W. R Wilmarth

P. W. Norris

T. L. Allen

MAY 29, 2007

Westinghouse Savannah River Company

Savannah River Site

Aiken, SC 29808

Prepared for the U.S. Department of Energy Under

Contract Number DE-AC09-96SR18500

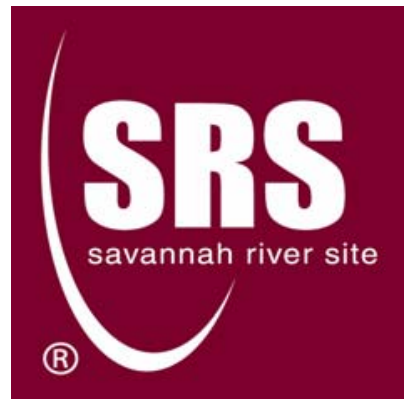




\section{DISCLAIMER}

This report was prepared for the United States Department of Energy under Contract No. DE-AC09-96SR18500 and is an account of work performed under that contract. Neither the United States Department of Energy, nor WSRC, nor any of their employees makes any warranty, expressed or implied, or assumes any legal liability or responsibility for accuracy, completeness, or usefulness, of any information, apparatus, or product or process disclosed herein or represents that its use will not infringe privately owned rights. Reference herein to any specific commercial product, process, or service by trade name, trademark, name, manufacturer or otherwise does not necessarily constitute or imply endorsement, recommendation, or favoring of same by Westinghouse Savannah River Company or by the United States Government or any agency thereof. The views and opinions of the authors expressed herein do not necessarily state or reflect those of the United States Government or any agency thereof.

Printed in the United States of America

Prepared For

U.S. Department of Energy 


\section{Approvals}

W. R. Wilmarth, Actinide and Chemical Technology

Date

P. W. Norris, Liquid Waste Operations

Date

T. L. Allen, Liquid Waste Operations

Date

D. J. Martin, Tank Farm Process Engineering

Date

A. M. Murray, Manager, Actinide and Chemical Technology

Date

Page 3 of 37 


\title{
RESULTS OF THE 2H EVAPORATOR ACID CLEANING AND IN-POT NEUTRALIZATION
}

\author{
W. R Wilmarth, P. W. Norris, and T. L. Allen \\ Washington Savannah River Company \\ Aiken, SC 29808
}

\section{SUMMARY}

The estimated 200 gallons of sodium aluminosilicate scale (NAS) present in the $242-16 \mathrm{H}$ Evaporator pot prior to chemical cleaning was subjected to four batches of $1.5 \mathrm{M}(9 \mathrm{wt} \%)$ nitric acid. Each batch was neutralized with 19 M (50 wt \%) sodium hydroxide (caustic) before transfer to Tank 38. The chemical cleaning process began on November 20, 2006, and was terminated on December 10, 2006. An inspection of the pot's interior was performed and based on data gathered during that inspection; the current volume of scale in the pot is conservatively estimated to be 36.3 gallons, which is well below the 200 gallon limit specified in the Technical Safety Requirements. In addition, the performance during all aspects of cleaning agreed well with the flowsheet developed at the bench and pilot scale. There were some lessons learned during the cleaning outage and are detailed in appendices of this report.

\section{INTRODUCTION}

The Savannah River Site (SRS) stores high level nuclear waste in 49 underground storage tanks. The wastes are to be vitrified in the Defense Waste Processing Facility (DWPF) for permanent disposal. The available tank space must be managed to ensure viability of the separation canyon to support nuclear material stabilization and continued operation of DWPF. Under normal operations, the wastes are evaporated to reduce volume. The SRS has three operational atmospheric-pressure high-level-waste evaporators. Two evaporators are located in H-Area and one is in F-Area. The 242-16H (or 2H) evaporator had not operated from October 1999 to September 2001 due to the presence of a large amount of sodium aluminosilicate scale that contained sodium diuranate. ${ }^{1,2,3}$ The scale is very similar to that observed in the aluminum and pulp paper industries ${ }^{4,5,6}$ and was produced at SRS by reaction of the aluminate supplied by the plutonium separations facilities and the silicate from recycle water from the DWPF. The chemistry of high level waste with elevated silicon levels thermodynamically favors the formation of aluminosilicates. ${ }^{7}$ The $2 \mathrm{H}$ Evaporator was scaled to the point that the concentrated evaporator bottoms could not be removed through normal steam lifting protocols.

Work performed by the Savannah River National Laboratory (SRNL) during calendar years 1998-2000 had shown that dilute nitric acid was an effective chemical cleaning agent. ${ }^{8,9}$ An overall cleaning flowsheet was developed in calendar year 2000 that addressed numerous safety issues associated with cleaning the pot, neutralizing the uranium-bearing acid and discharging the neutralized solutions to a waste tank. Beginning in May 2001, a depleted uranium and nitric acid mixture was added to the $2 \mathrm{H}$ Evaporator pot and heated to elevated temperatures. As a result of this action, the pot was cleaned and returned to service. 
As a result of the formation of aluminosilicates when elevated concentrations of silica are a concern, SRS changed the operational requirements for the site's High-Level Waste evaporators. Wastes containing high silicon concentrations, e.g., DWPF recycle, would be concentrated in the $2 \mathrm{H}$ Evaporator. The criticality hazard for the $2 \mathrm{H}$ Evaporator was reduced by depleting the U-235 content of the waste to below acceptable levels. Waste containing aluminate would be processed in the $2 \mathrm{~F}$ or $3 \mathrm{H}$ Evaporator and acceptance criteria were established to monitor for the possible formation of sodium aluminosilicate. ${ }^{10}$

Routine inspections of the $2 \mathrm{H}$ Evaporator pot have been performed periodically since the cleaning operations. In a recent inspection, evidence of scale growth has emerged. Additionally, difficulty in lifting the pot contents has been encountered along with a reduction in the pot siphon flowrates indicated an obstruction in the Gravity Drain Line (GDL). Hydro-lancing operations removed solid deposits from the GDL and samples were retrieved.

During 2005, SRNL, Liquid Waste Operations (LWO) and Planning Integration and Technology (PIT) personnel embarked on a program to develop a nitric acid-based flowsheet that would allow for in-pot neutralization of the spent acid prior to discharging to the waste tank, Tank $38 \mathrm{H}$. This work included dissolution testing of actual scale samples, ${ }^{11}$ neutralization of spent acid simulant containing uranium, ${ }^{12}$ and pilot-scale neutralization of a non-radioactive spent acid simulant in a one-tenth scale evaporator mockup. ${ }^{13,14}$ The result of this research and development effort was a flowsheet for implementation in the $242-16 \mathrm{H}$ Evaporator. ${ }^{15}$

As a result of successfully completing the research and development program, Liquid Waste Operations proceeded with an evaporator outage to perform the acid cleaning. Beginning on November 20, 2006, four acid treatments were completed. This report documents the cleaning results and provides discussion on the lessons learned throughout the outage.

\section{CLEANING OPERATIONS}

There are six basic steps in the cleaning operation and these are listed below:

- Acid Addition

- Heat up and dissolution

- Cooling

- Caustic addition and Neutralization

- Discharge to Tank 38

- Water Flush

The flowsheet utilized 1.5 M nitric acid to dissolve the aluminosilicates. Equation 1 shows the dissolution reactions for the major scale solids. The aluminosilicate reacts with nitric acid $\left(\mathrm{HNO}_{3}\right)$ to form sodium nitrate, aluminum nitrate and silicic acid $\left(\mathrm{H}_{2} \mathrm{SiO}_{3}\right)$. For simplicity, the waters of hydration are not included. Clarkeite $\left(\mathrm{Na}\left[\left(\mathrm{UO}_{2}\right) \mathrm{O}(\mathrm{OH})\right] \cdot \mathrm{H}_{2} \mathrm{O}\right)$ reacts with nitric acid to form sodium nitrate, uranyl nitrate $\left(\mathrm{UO}_{2}\left(\mathrm{NO}_{3}\right)_{2}\right)$, and water. Gibbsite $\left(\mathrm{Al}(\mathrm{OH})_{3}\right)$ reacts with nitric 
acid to form water and aluminum nitrate. The overall equations (from the crystalline phase to the solution phase) for dissolution of these three solids follow.

Equation 1. Dissolution Reactions for Primary Scale Solids with $\mathrm{HNO}_{3}$

$$
\begin{aligned}
\mathrm{Na}_{8} \mathrm{Al}_{6} \mathrm{Si}_{6} \mathrm{O}_{24}\left(\mathrm{NO}_{3}\right)_{2}+24 \mathrm{HNO}_{3} & \rightarrow 8 \mathrm{NaNO}_{3}+6 \mathrm{Al}\left(\mathrm{NO}_{3}\right)_{3}+6 \mathrm{H}_{2} \mathrm{SiO}_{3}+6 \mathrm{H}_{2} \mathrm{O} \\
\mathrm{Na}\left[\left(\mathrm{UO}_{2}\right) \mathrm{O}(\mathrm{OH})\right] \cdot \mathrm{H}_{2} \mathrm{O}+3 \mathrm{HNO}_{3} & \rightarrow \mathrm{NaNO}_{3}+\mathrm{UO}_{2}\left(\mathrm{NO}_{3}\right)_{2}+3 \mathrm{H}_{2} \mathrm{O} \\
\mathrm{Al}(\mathrm{OH})_{3}+3 \mathrm{HNO}_{3} & \rightarrow \mathrm{Al}\left(\mathrm{NO}_{3}\right)_{3}+3 \mathrm{H}_{2} \mathrm{O}
\end{aligned}
$$

Figure 1 and Figure 2 show data for a number of parameters from the $2 \mathrm{H}$ Evaporator pot during the first and second heating cycles. As observed in these figures, the rate of heating the pot is fairly rapid and reaching temperature $\left(\sim 90^{\circ} \mathrm{C}\right)$ within several hours. The pot pressure slowly rises and reaches about 4 in. water column. As expected, the specific gravity of the acid does not change as the scale dissolves. During heating the first batch of acid in the $2 \mathrm{H}$ Evaporator pot, the heating cycle was interrupted due to a cell sump alarm (see discussion in Appendix B and Data in Appendix C). Other than the sump issue, the other Batches 3 and 4 showed the same trends.

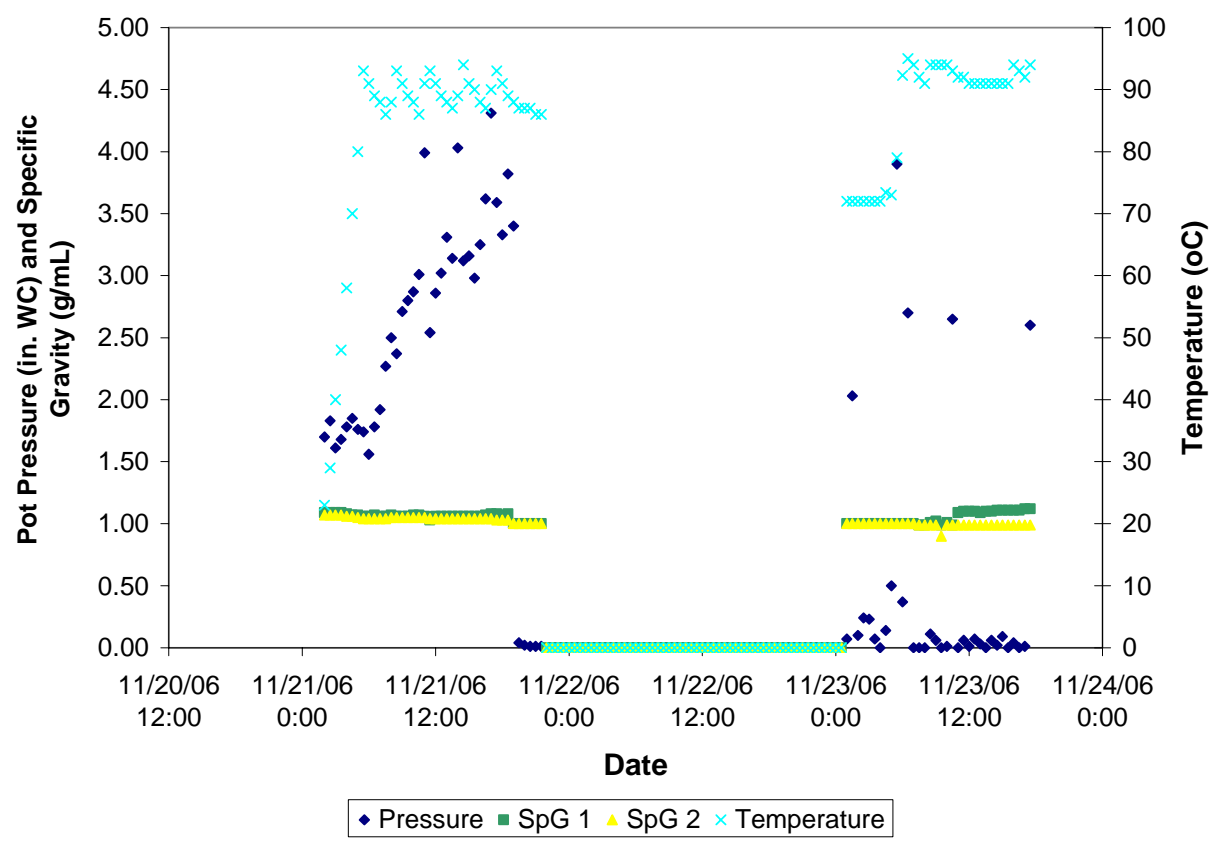

Figure 1. Plot of Data from Heating Pot during Batch 1 


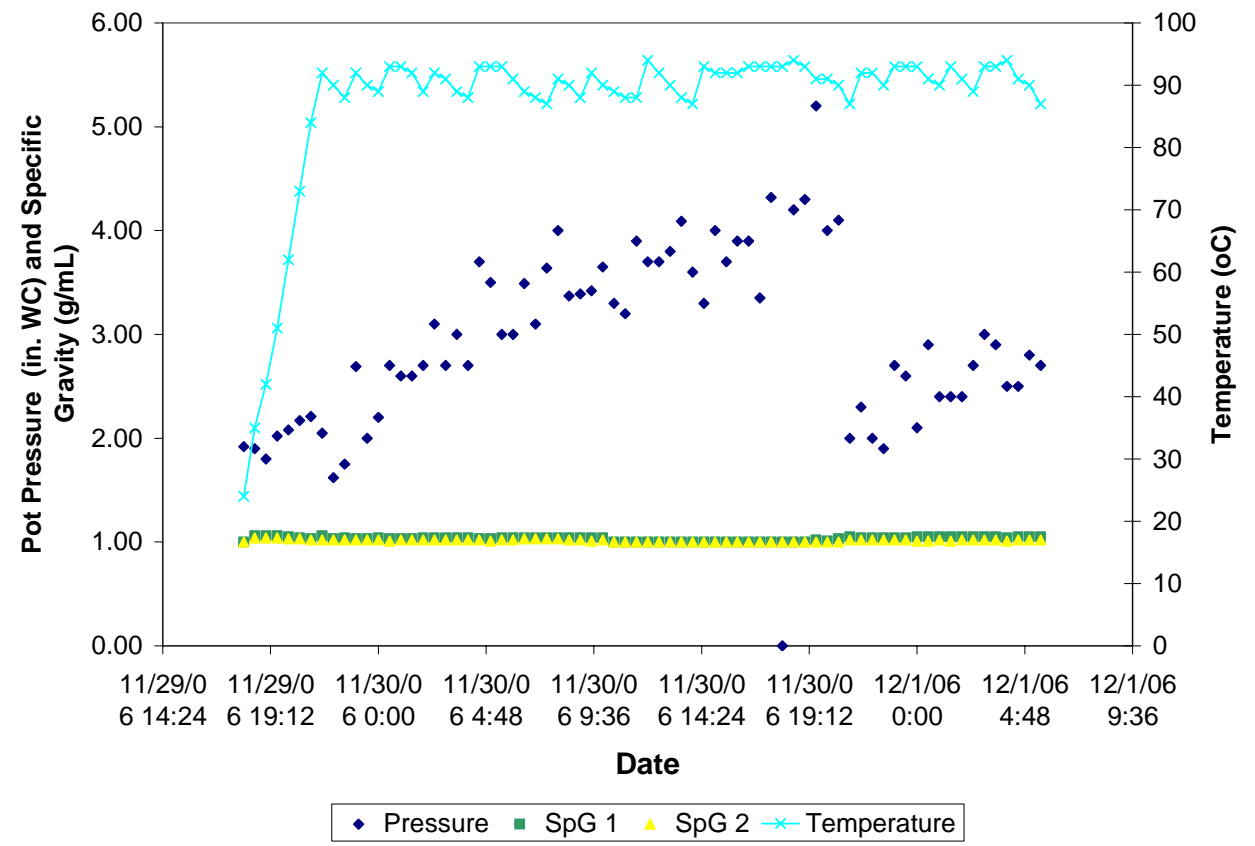

Figure 2. Data from Heating Pot during Batch 2

After the dissolution and heating cycle, the evaporator pot contents are cooled using water flow through the tube bundle. The contents are cooled to assist in reducing the maximum temperature reached during the neutralization step. This action is because of the exothermic reaction of the sodium hydroxide and nitric acid during neutralization. The desired effect will reduce the amount of aluminosilicate that reforms under the neutralized caustic composition. The cooling was modeled by Kwon ${ }^{16}$ in 2000 . Kwon used various calculational approaches to predict the length of time that it would take to reduce the evaporator pot temperature from the anticipated dissolution flowsheet temperature of $95{ }^{\circ} \mathrm{C}$ to $30^{\circ} \mathrm{C}$. The time required approximately 15 to 19 hours. In Figure 3, the cooling data are shown for the pot temperature for Batch 2. The duration observed agrees very well with the prediction and is well within the flowsheet duration of 32 hours. Batch 2 showed the longest time to cool to a nominal temperature of $30{ }^{\circ} \mathrm{C}$. The other batches cooled within $7-10$ hours. 


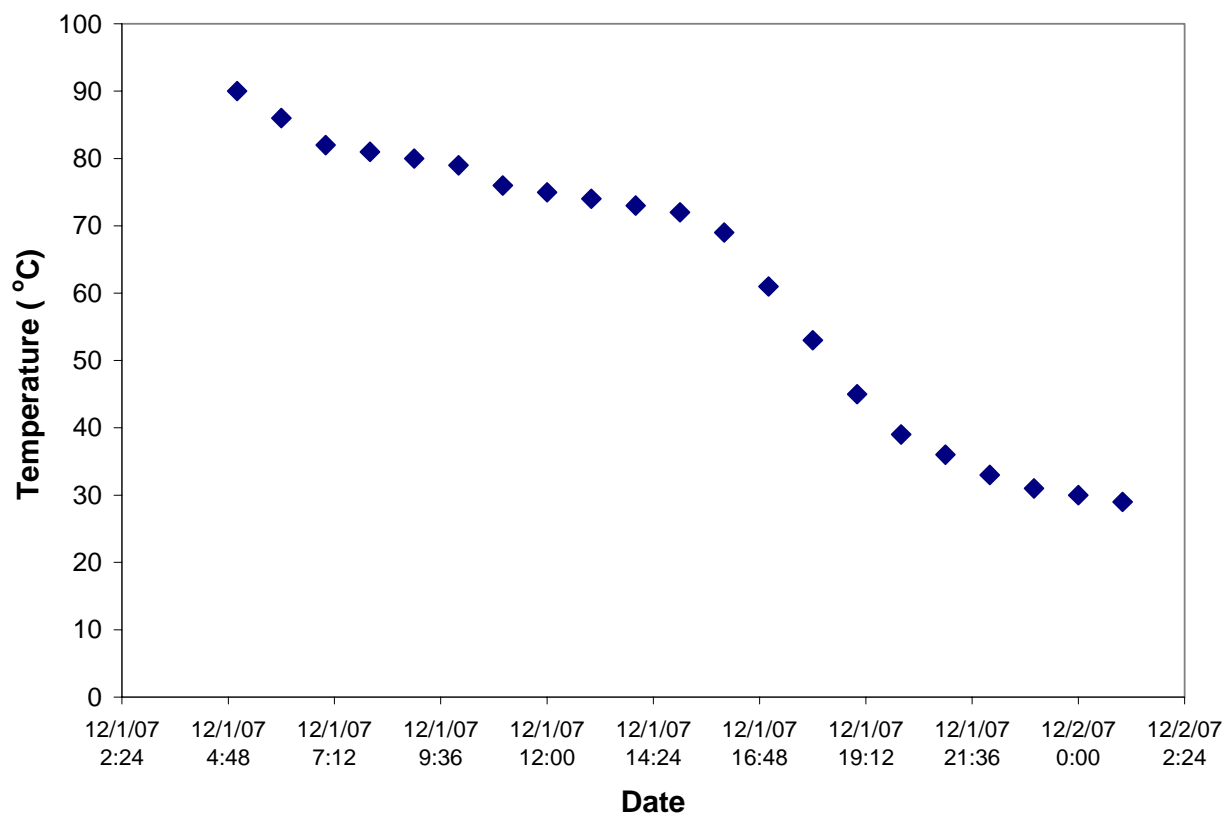

Figure 3. Cooling Curve for Batch 2

The spent acid solution cannot be added directly to a waste storage tank due to the potential for corrosion of the carbon steel by the acid, and is neutralized with sodium hydroxide prior to transfer to Tank $38 \mathrm{H}$. To ensure complete neutralization, excess sodium hydroxide is added to drive the $\mathrm{pH}$ to 14 reaching a free hydroxide concentration of approximately 1 molar.

Based on the products of the dissolution reactions shown, seven major chemical reactions occur during neutralization as shown in Equation 2.

\section{Equation 2. Neutralization Reactions for Dissolution Products With NaOH}

The following four reactions occur during neutralization to $\mathrm{pH} 7$.

$$
\begin{aligned}
\mathrm{HNO}_{3}+\mathrm{NaOH} & \rightarrow \mathrm{NaNO}_{3}+\mathrm{H}_{2} \mathrm{O} \\
\mathrm{Al}\left(\mathrm{NO}_{3}\right)_{3}+3 \mathrm{NaOH} & \rightarrow \mathrm{Al}(\mathrm{OH})_{3}+3 \mathrm{NaNO}_{3} \\
\mathrm{UO}_{2}\left(\mathrm{NO}_{3}\right)_{2}+2 \mathrm{NaOH} & \rightarrow \mathrm{UO}_{2}(\mathrm{OH})_{2}+2 \mathrm{NaNO}_{3} \\
\mathrm{H}_{2} \mathrm{SiO}_{3}+\mathrm{NaOH} & \rightarrow \mathrm{NaHSiO}_{3}+\mathrm{H}_{2} \mathrm{O}
\end{aligned}
$$

The following three reactions occur during adjustment to $\mathrm{pH}>13$.

$$
\begin{aligned}
\mathrm{Al}(\mathrm{OH})_{3}+\mathrm{NaOH} & \rightarrow \mathrm{NaAlO}_{2}+2 \mathrm{H}_{2} \mathrm{O} \\
2 \mathrm{UO}_{2}(\mathrm{OH})_{2}+2 \mathrm{NaOH} & \rightarrow \mathrm{Na}_{2} \mathrm{U}_{2} \mathrm{O}_{7}+3 \mathrm{H}_{2} \mathrm{O} \\
\mathrm{NaHSiO}_{3}+\mathrm{NaOH} & \rightarrow \mathrm{Na}_{2} \mathrm{SiO}_{3}+\mathrm{H}_{2} \mathrm{O}
\end{aligned}
$$


Figure 4 shows the measured evaporator pot parameters for the neutralization step. After the evaporator pot contents had cooled to approximately $30^{\circ} \mathrm{C}$, concentrated sodium hydroxide solution $(50 \mathrm{wt} \%)$ is added to the $2 \mathrm{H}$ Evaporator pot with agitation provided by the air sparger. One can observe that the sodium hydroxide addition raised the volume of the pot from $\sim 47$ inches to 60 inches. The caustic addition was performed using two totes of $50 \mathrm{wt} \%$ sodium hydroxide as evidenced by the two step change in pot volume. The temperature rose from $23{ }^{\circ} \mathrm{C}$ to $39^{\circ} \mathrm{C}$ following the first tote addition. The data show that during the change out of the totes the temperature fell below $30^{\circ} \mathrm{C}$ and rose only slightly during the second tote addition. The pot pressure was constant as expected. The specific gravity measurements differed with the lower tube showing an increase in specific gravity from 1.12 to $1.20 \mathrm{~g} / \mathrm{mL}$. The upper measurement did not show this increase. All of these trends were repeated in the other neutralization batches. The operation went very smoothly.

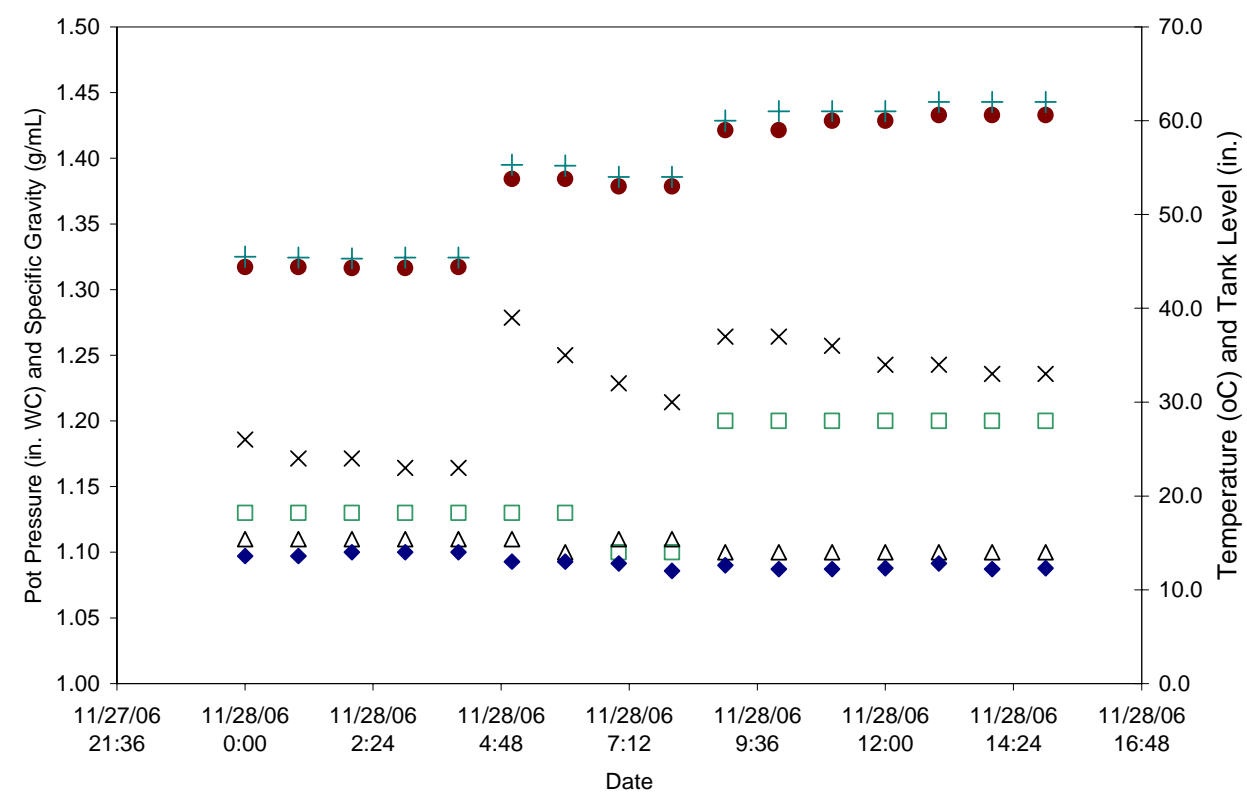

$\square$ SpG $1 \Delta$ SpG $2 \bullet$ Pressure $\times$ Temperature $\bullet$ Liquid Level $1+$ Liquid Level 2

Figure 4. Data from Neutralization of Batch 1

\section{RESULTS AND DISCUSSION}

After two dissolution batches were performed, a video inspection was performed. The results indicated that the scale appeared to be dissolving slowly. As shown in the picture in Figure 5, the picture on the right shows some material still adhering to the wall of the pot. This picture looks similar to a picture taken from the pot in 2001 shown in Figure 5 on the left. In the cleaning that occurred in 2001, it was estimated that there were $3500 \mathrm{~kg}$ of scale in the pot. ${ }^{17}$ Analysis of the data in 2001 showed only $350 \mathrm{~kg}$ had dissolved. ${ }^{18}$ 
This information implies that during the dissolution the scale was removed from the walls and piping and was removed from the pot by the pump rather than dissolution. It appears that in the 2006 dissolution campaign, the scale is adhering to the walls more than in 2001. This result is a similar behavior as that of the Gravity Drain Line. One aspect to remember is that in 2001, the scale formed in a rapid occurrence. The scale currently in the pot has been growing slowly and has been aged in place by the number of heating and cooling cycles. Additional acid treatment should further dissolve the scale.

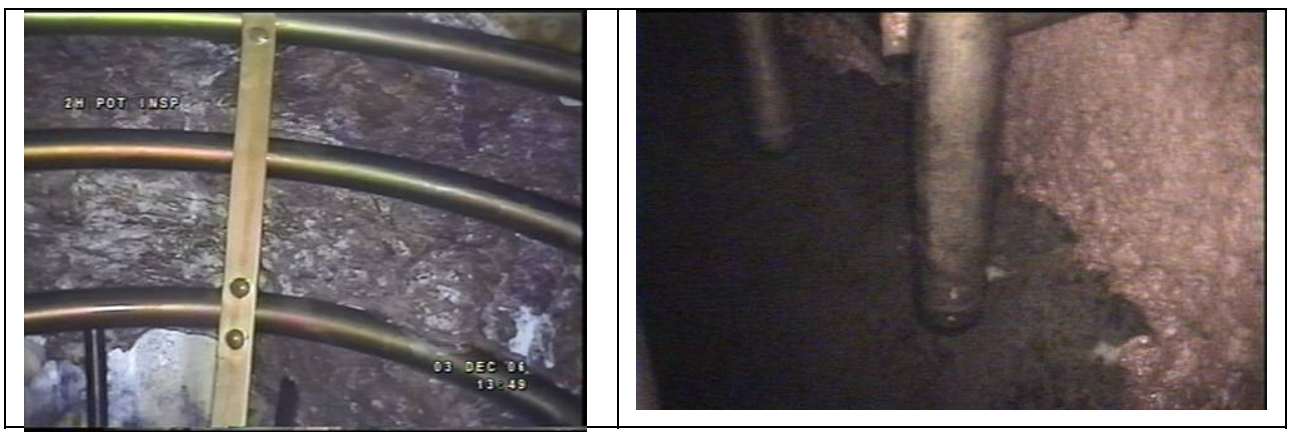

Figure 5. Partially Dissolved Scale

Figure 6 shows a photograph of the tube bundle and warming coil. A golden appearance is observed that is most probably an artificially induced by the lighting in conjunction with the digital photography that is now being utilized. SRNL did not see anything that would indicate corrosion or other concern. As shown in Figure 6, the color of the tube bundle changes significantly as one move from the left lower portion to the upper right portion.

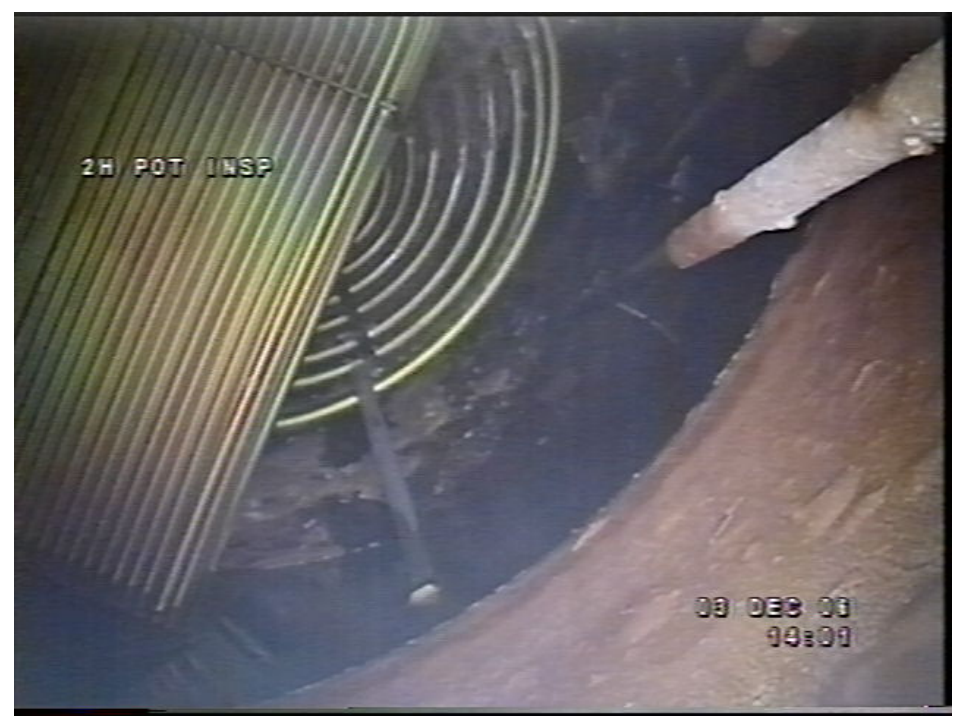

Figure 6. Photograph of the Tube Bundle and Warming Coils 
Figure 7 shows a portion of a photograph of the $2 \mathrm{H}$ Evaporator pot. SRNL was asked to comment on the nature of the material above the liquid level that had been cleaned. As we can not precisely know what the material is, we compared the nature of the material to the photographs that have been taken from the pot and GDL over time. Our opinion is that material likely contains sodium aluminosilicate scale. To reduce the salt content of this film, one could envision filling the pot with water, bringing it to a boil, then slowly raising the liquid level to as near the demister as possible to allow the hot water to dissolve the salt.

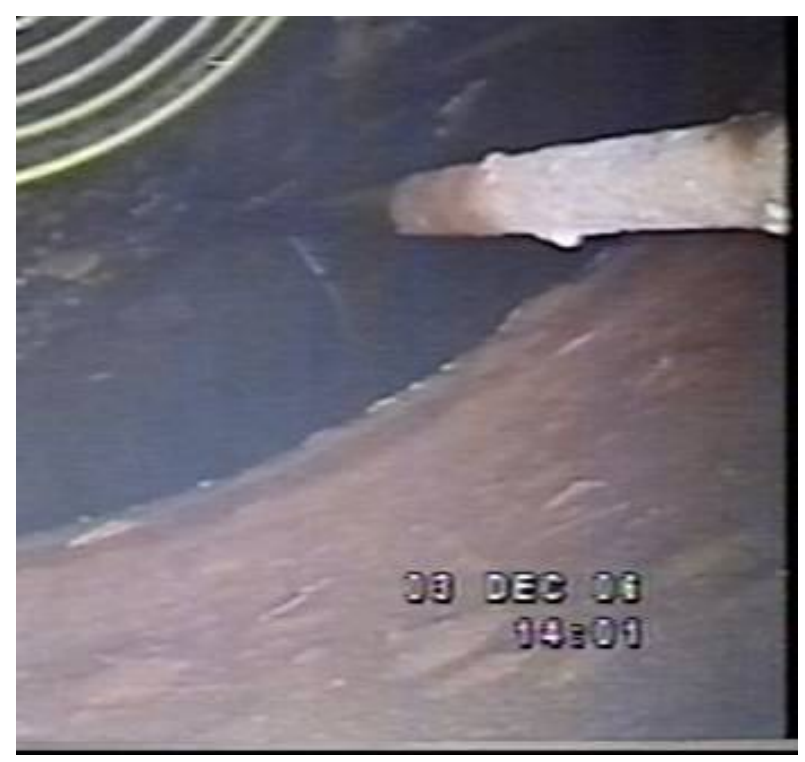

Figure 7. Photograph of Solids in the Vapor Space of the $2 \mathrm{H}$ Evaporator Pot

Two additional acid strikes were performed with their accompanying neutralizations and discharges to Tank $38 \mathrm{H}$. The results of the video inspections of the pot following completion of acid cleaning showed that the majority of the scale dissolved and was removed. However, there was one spot within the pot that was rather resistant to the nitric acid treatment. This spot is shown in Figure 8. A small amount of scale remained between the wall and the warming coil.

To restart the evaporator for waste service, LWO personnel estimated the amount of scale remaining in the $2 \mathrm{H}$ Evaporator pot. This estimate is performed using the digital photography and video evidence and comparing this evidence of the know design of the evaporator pot. Shown in Figure 9 is depiction of the area of the evaporator where scale remains between the wall and the warming coil. Knowing the dimensions of the warming coil tubing and the distance between the coil and wall, the volume of scale can be estimated. Additionally, the bottom of the pot was not visible and was assumed to contain aluminosilicate scale. LWO engineering estimated 36.3 gallons of scale which is well below the limit of 200 gallons. Resumption of waste evaporation began shortly thereafter. 


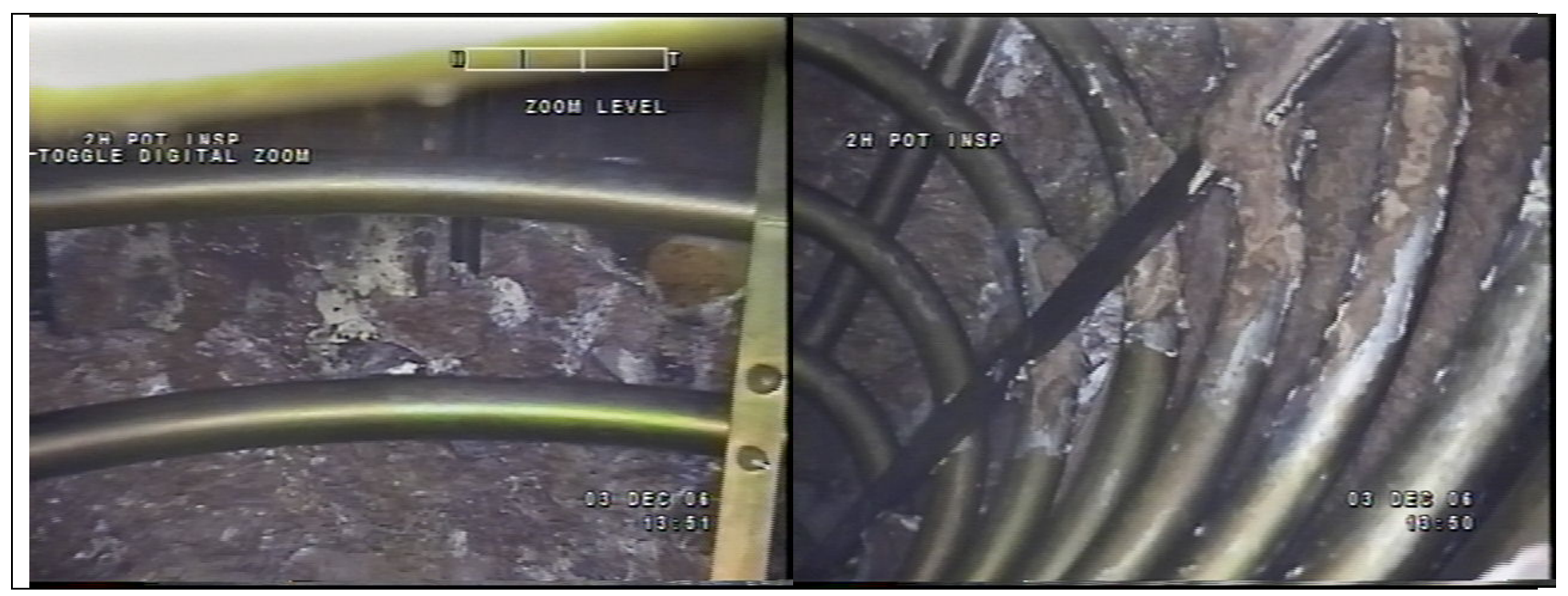

Figure 8. Scale Remnants in $2 \mathrm{H}$ Pot following Acid Cleaning

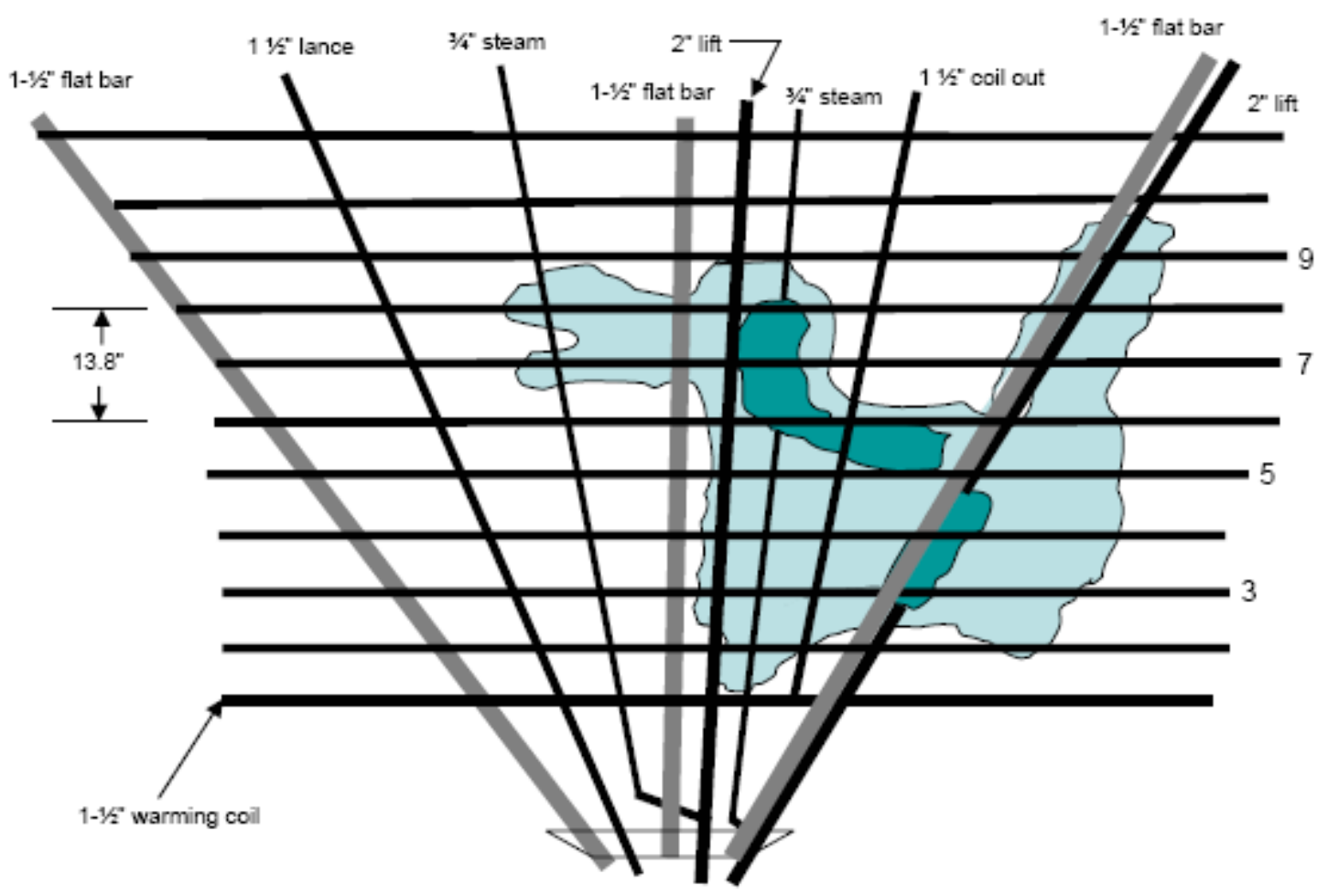

Figure 9. Depiction of Remaining Scale in $2 \mathrm{H}$ following Acid Cleaning 


\section{CONCLUSIONS}

Processing of DWPF recycle through the $2 \mathrm{H}$ Evaporator will continue to generate the formation of sodium aluminosilicate scale due the large quantities of aluminum in the liquor and the saltcake and the legacy of silicon in the feed and drop tanks. This formation will continue until one of these two elements ( $\mathrm{Al}$ and $\mathrm{Si}$ ) is depleted. Therefore, sodium aluminosilicate scale will continue to deposit and acid cleaning will be necessary.

The intent of the latest flowsheet development and deployment was to show that acid cleaning followed by in-pot neutralization would successfully reduce the amount of scale efficiently. The results of the research and development, the facility design, and the facility procedures and operation collectively show success. This flowsheet deployment showed a steady and predictable dissolution of the scale. The facility installation and removal proved to be facile and can be re-installed as needed for future cleaning operations. 


\section{APPENDIX A. SHIFT MANAGER'S LOG ENTRIES}

Log Entries from Shift Manager's Log Book

\begin{tabular}{|c|c|c|c|}
\hline Date & Time & Activity & Source \\
\hline \multirow[t]{4}{*}{$11 / 20 / 06$} & $16: 50$ & Acid being added to pot. & SM \\
\hline & $17: 40$ & $\begin{array}{l}\text { Pump flow startup rate issue. Caused over flow of funnel. Flow } \\
\text { rate } 5 \text { to } 50 \mathrm{gpm} \text {. }\end{array}$ & SM \\
\hline & $22: 30$ & Pumped acid into pot at $25 \mathrm{gpm}$. & SM \\
\hline & $23: 37$ & Completed adding 2000 gallons of nitric acid. & SM \\
\hline \multirow[t]{4}{*}{$11 / 21 / 06$} & 02:00 & Placed steam on the tube bundle. & SM \\
\hline & $05: 23$ & Started 32 hour simmer clock. & SM \\
\hline & $18: 56$ & Completed shutdown of $2 \mathrm{H}$ Evaporator. & SM \\
\hline & 20:00 & Issue with cell sump ARP. & SM \\
\hline $11 / 22 / 06$ & $20: 30$ & Completed adding caustic to $2 \mathrm{H}$ evap cell sump. & SM \\
\hline \multirow[t]{5}{*}{$11 / 23 / 06$} & $00: 53$ & Steam placed on $2 \mathrm{H}$ evap tube bundle. & SM \\
\hline & $05: 38$ & Reached $85^{\circ} \mathrm{C}$ in $2 \mathrm{H}$ evap pot Simmer time begins now. & SM \\
\hline & $17: 10$ & Completed $2 \mathrm{H}$ "Acid cleaning simmer time" & SM \\
\hline & $20: 16$ & Flushed dip tubes. & SM \\
\hline & $20: 50$ & Placed WW in $2 \mathrm{H}$ evap tube bundle. Current temp $82^{\circ} \mathrm{C}$. & SM \\
\hline \multirow[t]{3}{*}{$11 / 24 / 06$} & $00: 35$ & Current pot temp is $43^{\circ} \mathrm{C}$ & SM \\
\hline & 03:40 & $\begin{array}{l}\text { Problem with proper air flow for agitation. Current pot temp is } \\
30^{\circ} \mathrm{C} \text {. }\end{array}$ & SM \\
\hline & 09:30 & $\begin{array}{l}\text { Reworked pressure regulator. Replaced filter. Replaced globe } \\
\text { valves with gate valves. Replaced with larger compressor. } \\
\text { Finally realized that the flow meter scale was not proper calibrated } \\
\text { for the actual conditions. }\end{array}$ & SM \\
\hline \multirow[t]{6}{*}{$11 / 28 / 06$} & $04: 15$ & Started adding first caustic tote. & SM \\
\hline & $04: 55$ & Completed adding the first caustic tote. & SM \\
\hline & $08: 16$ & Started adding second caustic tote. & SM \\
\hline & $08: 45$ & Completed adding second caustic tote. & SM \\
\hline & $21: 14$ & Initiated pumping 2H Evaporator pot to Tk 38. & SM \\
\hline & $21: 48$ & Completed pumping $2 \mathrm{H}$ Evaporator pot to Tk 38. & $\mathrm{SM}$ \\
\hline \multirow[t]{4}{*}{$11 / 29 / 06$} & $15: 15$ & Initiated acid addition to $2 \mathrm{H}$ pot. & SM \\
\hline & $17: 23$ & Completed flushing of acid addition lines@2H Evap. & SM \\
\hline & $18: 00$ & Initiated heating of acid in $2 \mathrm{H}$ Pot. & SM \\
\hline & $22: 22$ & Initiated 32 hour simmer time for $2 \mathrm{H}$ Evaporator. The pot temp is & $\mathrm{SM}$ \\
\hline
\end{tabular}




\begin{tabular}{|c|c|c|c|}
\hline Date & Time & Activity & Source \\
\hline & & $90^{\circ} \mathrm{C}$. The simmer will be complete on 12/1/05@5:22 & \\
\hline $11 / 30 / 06$ & 21:04 & Completed flush of $2 \mathrm{H}$ Evaporator dip tubes using 38 gallons. & $\mathrm{SM}$ \\
\hline \multirow[t]{2}{*}{$12 / 1 / 06$} & $05: 30$ & $\begin{array}{l}\text { Completed } 32 \text { hour simmer of } 2 \mathrm{H} \text { Evaporator pot with nitric acid. } \\
\text { Secured steam to the pot. }\end{array}$ & SM \\
\hline & $16: 17$ & $\begin{array}{l}\text { Placed cooling water on } 2 \mathrm{H} \text { tube bundle. Current pot temperature } \\
79^{\circ} \mathrm{C} \text {. }\end{array}$ & SM \\
\hline \multirow[t]{13}{*}{$12 / 2 / 06$} & $02: 15$ & Cam lok fitting on caustic tote discharge leaked. & SM \\
\hline & $02: 38$ & $\begin{array}{l}\text { Started caustic addition to } 2 \mathrm{H} \text { Evap pot; } 50.8 \text { in indicated evap pot } \\
\text { level. Temp } 27^{\circ} \mathrm{C} \text {. }\end{array}$ & SM \\
\hline & $03: 25$ & Completed addition of $1^{\text {st }}$ caustic tote to $2 \mathrm{H}$ evap pot. & SM \\
\hline & 04:19 & Started addition of $2^{\text {nd }}$ caustic addition of $2^{\text {nd }}$ caustic tote. & SN \\
\hline & 05:02 & Completed addition of $2^{\text {nd }}$ caustic tote. & SM \\
\hline & $10: 25$ & Completed pulling $\mathrm{OH} \# 1$ sample at $2 \mathrm{H}$. & SM \\
\hline & $16: 40$ & Shutdown will water to $2 \mathrm{H}$ tube bundle. & SM \\
\hline & 17:01 & Started pumping to $\mathrm{Tk} 38$. & SM \\
\hline & $17: 25$ & Completed emptying $2 \mathrm{H}$ pot. & $\mathrm{SM}$ \\
\hline & $17: 50$ & Completed pumping out $2 \mathrm{~h} \mathrm{OH} \mathrm{Tk \# 1.}$ & SM \\
\hline & $21: 55$ & Started well water addition to $2 \mathrm{H}$ Evaporator pot to $\sim 98$ inches. & SM \\
\hline & $22: 53$ & $\begin{array}{l}2 \mathrm{H} \text { evap pot level reached } 95 \text { inches stopped well water addition } \\
\text { to evap pot. }\end{array}$ & $\mathrm{SM}$ \\
\hline & $23: 32$ & Started pumping down $2 \mathrm{H}$ evap to Tk 38. Initial level 95 inches. & $\mathrm{SM}$ \\
\hline \multirow[t]{3}{*}{$12 / 3 / 06$} & $00: 27$ & Completed pump down rate is $\sim 81 \mathrm{gpm}$. & SM \\
\hline & $12: 00$ & Started 2H Pot inspection between light rains. & SM \\
\hline & $14: 10$ & $\begin{array}{l}\text { Completed } 2 \mathrm{H} \text { pot inspection. Still see some scaling on the } \\
\text { warming coils and the cone. }\end{array}$ & SM \\
\hline $12 / 4 / 06$ & $21: 15$ & $\begin{array}{l}\text { Started adding nitric acid at } 2 \mathrm{H} \text { evap pot. Flow is being raised to } \\
\text { a target of } 20 \mathrm{gpm} \text {. }\end{array}$ & $\mathrm{SM}$ \\
\hline \multirow[t]{2}{*}{$12 / 5 / 06$} & $00: 50$ & $\begin{array}{l}\text { Initiated steam to } 2 \mathrm{H} \text { evaporator pit tube bundle. Existing } \\
\text { temperature } 17^{\circ} \mathrm{C} \text {. }\end{array}$ & $\mathrm{SM}$ \\
\hline & $4: 10$ & $\begin{array}{l}2 \mathrm{H} \text { evap pot reached } 85^{\circ} \mathrm{C} \text {. Start } 32 \mathrm{hr} \text { clock for simmer }(1210 \\
12 / 6 / 06) \text {. }\end{array}$ & $\mathrm{SM}$ \\
\hline \multirow[t]{3}{*}{$12 / 6 / 06$} & $13: 10$ & $\begin{array}{l}\text { Notified that the } 2 \mathrm{H} \text { Evaporator TCV is now full open supplying } \\
\text { cooling water to tube bundle. Current temp is } 84^{\circ} \mathrm{C} \text {. }\end{array}$ & $\mathrm{SM}$ \\
\hline & $22: 25$ & Started adding caustic to $2 \mathrm{H}$ Evap. & $\mathrm{SM}$ \\
\hline & $22: 50$ & Completed adding the $1^{\text {st }}$ caustic tote in $2 \mathrm{H}$ Evap. & SM \\
\hline $12 / 7 / 06$ & $01: 25$ & Completed adding $2^{\text {nd }}$ tote to $2 \mathrm{H}$ Evap. The $10 \mathrm{hr}$ agitation will be & SM \\
\hline
\end{tabular}




\begin{tabular}{|c|c|c|c|}
\hline Date & Time & Activity & Source \\
\hline & & completed at 1125 . & \\
\hline & $23: 28$ & Placed steam on $2 \mathrm{H}$ to start heatup of the pot. Current temp $28^{\circ} \mathrm{C}$. & SM \\
\hline \multirow[t]{4}{*}{$12 / 8 / 06$} & 02:38 & $\begin{array}{l}2 \mathrm{H} \text { evap pot reached } 85^{\circ} \mathrm{C} .32 \text { hour simmer started. Time will be } \\
\text { up at } 1038 \text {. }\end{array}$ & SM \\
\hline & $15: 15$ & $\begin{array}{l}2 \mathrm{H} \mathrm{CRO} \mathrm{reported,} \mathrm{while} \mathrm{trying} \mathrm{to} \mathrm{adjust} \mathrm{steam} \mathrm{to} \mathrm{the} \mathrm{tube} \mathrm{bundle,} \\
\text { they noticed that the AIV had closed. The tried to reset. Appears } \\
\text { there is no power. Call E\&I to trouble shoot. Current temp } 89^{\circ} \mathrm{C} \text {. }\end{array}$ & SM \\
\hline & 05:40 & $\begin{array}{l}\text { E\&I found that the breaker may be bad. E\&I opened the close } \\
\text { breaker and power came back on. Power supplied from HM-242- } \\
\text { 16H-ELLVIPNL-LV-3 Breaker } 3 \text {. }\end{array}$ & SM \\
\hline & 07:30 & E\&I to investigate 2H evap AIV breaker at LP-3 w/o 732923. & SM \\
\hline \multirow[t]{5}{*}{$12 / 9 / 06$} & 04:31 & $\begin{array}{l}\text { Simmering of acid @ 2H evap. pot continues until } 1040 \text { hr this } \\
\text { morning. }\end{array}$ & SM \\
\hline & $10: 50$ & Completed $32 \mathrm{hr}$ simmer at $2 \mathrm{H}$ evaporator between $85-95 \mathrm{C}$. & SM \\
\hline & 14:10 & $\begin{array}{l}\text { Well water flow has been initiated to the } 2 \mathrm{H} \text { tube bundle for cool } \\
\text { down. Starting temp was 77C @ } 1332 \text { and current temp is } 71 \mathrm{C} \text {. }\end{array}$ & SM \\
\hline & $21: 52$ & Initiated caustic addition to $2 \mathrm{H}$ pot. & SM \\
\hline & 23:50 & Completed emptying $1^{\text {st }}$ caustic tote ( $\sim 247$ gallons). & SM \\
\hline \multirow[t]{8}{*}{$12 / 10 / 06$} & 00:48 & $\begin{array}{l}\text { Completed addition of } 2^{\text {nd }} \text { caustic tote to } 2 \mathrm{H} \text { evap. pot level is } \\
\text { @ } 71 \text { " - total caustic addition to pot is } 434 \text { gallons. }\end{array}$ & SM \\
\hline & $11: 57$ & Secured cooling wtr to $2 \mathrm{H}$ evaporator tube bundle. & SM \\
\hline & 12:18 & Started pumping neutralization pot contents to Tk 38. & SM \\
\hline & $12: 53$ & Completed emptying of $2 \mathrm{H}$ evap pot to Tk 38. & SM \\
\hline & 13:12 & Started filling $2 \mathrm{H}$ evap pot for well water flush. & SM \\
\hline & 13:45 & Completed WW addition to $2 \mathrm{H}$ pot $91 ”$. & SM \\
\hline & 13:53 & Started pump out of $2 \mathrm{H}$ evap pot. & SM \\
\hline & $14: 32$ & $\begin{array}{l}\text { Completed pump out of } 2 \mathrm{H} \text { evap pot. Performing Chem Clean } \\
\text { Shutdown. }\end{array}$ & SM \\
\hline \multirow[t]{7}{*}{$12 / 11 / 06$} & 10:06 & Pulled pug for pot inspection. & SM \\
\hline & $11: 35$ & Completed pot inspection. & SM \\
\hline & 16:08 & Started adding the first caustic tote. & SM \\
\hline & $16: 38$ & Completed adding the $1^{\text {st }}$ caustic tote to $2 \mathrm{H}$ potl. & SM \\
\hline & 19:35 & $\begin{array}{l}\text { Completed adding } 2 \text { totes for caustic totes ( } 521 \text { gallons) } \\
\text { Completed flushing of caustic }\end{array}$ & SM \\
\hline & 20:07 & Completed dip tube flush@2H evap. (28 gals). & SM \\
\hline & $20: 25$ & $2 \mathrm{H}$ tube bundle covered with IW. (Really flush/well water.) & SM \\
\hline
\end{tabular}




\begin{tabular}{|c|c|l|c|}
\hline Date & Time & \multicolumn{1}{|c|}{ Activity } & Source \\
\hline & $20: 44$ & Placed steam on to tube bundle. & SM \\
\hline $12 / 12 / 07$ & $04: 30$ & Shutdown steam to tube bundle @ 2H evap. & SM \\
\hline & & & \\
\hline
\end{tabular}

Log Entries from 28H Control Room Log

\begin{tabular}{|c|c|c|c|}
\hline Date & Time & Activity & Source \\
\hline \multirow[t]{7}{*}{$11 / 20 / 06$} & $14: 13$ & Initiating Sect 7.12 (Adding Nitric Acid to the Evap Pot) & $\begin{array}{c}28 \mathrm{H} \\
\mathrm{CR}\end{array}$ \\
\hline & $16: 15$ & Drain Valve to Tanker opened. & $\begin{array}{c}28 \mathrm{H} \\
\mathrm{CR}\end{array}$ \\
\hline & $16: 23$ & $\begin{array}{l}\text { Pump shutdown due to splash at funnel; } 1-2 \text { gals on apron; no } \\
\text { personnel injury; placing system in safe condition; do not meet } \\
\text { entry requirements into AOP-005 due to containment in apron. }\end{array}$ & $\begin{array}{c}28 \mathrm{H} \\
\mathrm{CR}\end{array}$ \\
\hline & $22: 07$ & Nitric acid tanker drain valves opened. & $\begin{array}{c}28 \mathrm{H} \\
\mathrm{CR}\end{array}$ \\
\hline & $22: 09$ & Nitric acid pump started per section 7.12 . & $\begin{array}{l}28 \mathrm{H} \\
\mathrm{CR}\end{array}$ \\
\hline & $22: 17$ & $\begin{array}{l}\text { Increased nitric acid flow from } 10 \mathrm{gpm} \text { to } 15 \mathrm{gpm} \text {. No leaks or } \\
\text { overflow observed. }\end{array}$ & $\begin{array}{c}28 \mathrm{H} \\
\mathrm{CR}\end{array}$ \\
\hline & $23: 37$ & $\begin{array}{l}\text { Shutdown Nitric acid pump \& closed NAS-V-7. Evaporator pot } \\
\text { level now @61.0" per totalizer reading, 2000 gals nitric acid } \\
\text { added/transferred, per conversion table 6.2, } 2227 \text { gallons nitric } \\
\text { a received into pot, difference }=227 \text { gals. }\end{array}$ & $\begin{array}{c}28 \mathrm{H} \\
\mathrm{CR}\end{array}$ \\
\hline \multirow[t]{7}{*}{$11 / 21 / 06$} & 00:05 & Began flush of nitric acid addition system per section 7.20 . & $\begin{array}{c}28 \mathrm{H} \\
\mathrm{CR}\end{array}$ \\
\hline & 01:07 & $\begin{array}{l}\text { Completed section } 7.20 \text {, Flush of Nitric Acid Addition System, } \\
50 \text { gals flush water used. }\end{array}$ & $\begin{array}{c}28 \mathrm{H} \\
\mathrm{CR}\end{array}$ \\
\hline & $01: 13$ & Initiated heating evap pot per section 4.4 & $\begin{array}{c}28 \mathrm{H} \\
\mathrm{CR}\end{array}$ \\
\hline & 02:00 & $\begin{array}{l}\text { Placed steam on tube bundle per Heating of Evap Pot section } \\
\text { 4.4. Initial tube bundle steam flow @ } 11 \mathrm{lb} / \mathrm{hr} \text {. }\end{array}$ & $\begin{array}{c}28 \mathrm{H} \\
\mathrm{CR}\end{array}$ \\
\hline & 03:00 & $\begin{array}{l}\text { Increased tube bundle steam flow from } 11 \mathrm{lbm} / \mathrm{hr} \text { to } 100 \\
\mathrm{lbm} / \mathrm{hr} \text {. }\end{array}$ & $\begin{array}{c}28 \mathrm{H} \\
\mathrm{CR}\end{array}$ \\
\hline & 04:01 & $\begin{array}{l}\text { Increased tube bundle steam flow to } 200 \mathrm{lbm} / \mathrm{hr} \text {, evap pot temp } \\
\text { @ } 61^{\circ} \mathrm{C} \text {. }\end{array}$ & $\begin{array}{c}28 \mathrm{H} \\
\mathrm{CR}\end{array}$ \\
\hline & $05: 02$ & $\begin{array}{l}\text { Increased tube bundle steam flow to } 300 \mathrm{lbm} / \mathrm{hr} \text {, evap pot temp } \\
\text { (a) } 80^{\circ} \mathrm{C} \text {. }\end{array}$ & $\begin{array}{l}28 \mathrm{H} \\
\mathrm{CR}\end{array}$ \\
\hline
\end{tabular}


WSRC-STI-2007-00286, REVISON 0

\begin{tabular}{|c|c|c|c|}
\hline \multirow[t]{5}{*}{ Date } & Time & Activity & Source \\
\hline & $05: 23$ & $\begin{array}{l}\text { Evap pot temp now @ } 90^{\circ} \mathrm{C} . \mathrm{S} / \mathrm{M} \text { notified. Simmering period } \\
\text { has begun. }\end{array}$ & $\begin{array}{l}28 \mathrm{H} \\
\mathrm{CR}\end{array}$ \\
\hline & $08: 15$ & $\begin{array}{l}\text { Received “CRC FEED TK HI-HI LEVEL CONDUCTIVITY”. } \\
\text { Responded per ARP. }\end{array}$ & $\begin{array}{c}28 \mathrm{H} \\
\mathrm{CR}\end{array}$ \\
\hline & $18: 37$ & $\begin{array}{l}\text { Received Evap sump HI LVL COND PROBE; entered 3.7.12 } \\
\text { Cond A; Notified Shift Manager. }\end{array}$ & $\begin{array}{c}28 \mathrm{H} \\
\mathrm{CR}\end{array}$ \\
\hline & $18: 56$ & $\begin{array}{l}\text { Completed shutdown per } 4.4 \text { of Chemical Cleaning, due to } \\
\text { Evap cell sump cond. probe. }\end{array}$ & $\begin{array}{c}28 \mathrm{H} \\
\mathrm{CR}\end{array}$ \\
\hline \multirow[t]{4}{*}{$11 / 22 / 06$} & $04: 30$ & Pump Overheads tank \#2 to ETP. & $\begin{array}{c}28 \mathrm{H} \\
\mathrm{CR}\end{array}$ \\
\hline & $14: 06$ & $\begin{array}{l}\text { E\&I completed repairs to CRC Feed Tank level loop - low } \\
\text { level alarm that had been interlocking pump is clear. }\end{array}$ & $\begin{array}{c}28 \mathrm{H} \\
\mathrm{CR}\end{array}$ \\
\hline & $21: 10$ & $\begin{array}{l}\text { Completed caustic addition and sampling of Evap cell sump, } \\
\text { results are approx pH-12.0. }\end{array}$ & $\begin{array}{c}28 \mathrm{H} \\
\mathrm{CR}\end{array}$ \\
\hline & $23: 20$ & $\begin{array}{l}\text { Completed jetting Evap cell sump to Tk } 43 \text {, jetted a total of } 315 \\
\text { gallons to Tk } 43 \text {. }\end{array}$ & $\begin{array}{c}28 \mathrm{H} \\
\mathrm{CR}\end{array}$ \\
\hline \multirow[t]{4}{*}{$11 / 23 / 06$} & $00: 53$ & Began heating Evap pot per Sec 4.4 of Chem/Cleaning manual. & $\begin{array}{c}28 \mathrm{H} \\
\mathrm{CR}\end{array}$ \\
\hline & $05: 38$ & $\begin{array}{l}\text { Reached } 85^{\circ} \mathrm{C} \text { on the Evap pot temp and began the } 11.5 \mathrm{HR} \\
\text { simmer time. }\end{array}$ & $\begin{array}{c}28 \mathrm{H} \\
\mathrm{CR}\end{array}$ \\
\hline & $17: 10$ & $\begin{array}{l}\text { Shutdown Evaporator Pot Heating AIV Closed and Lance GV } \\
\text { in AIR } 32 \text { hours heating with acid complete. }\end{array}$ & $\begin{array}{c}28 \mathrm{H} \\
\mathrm{CR}\end{array}$ \\
\hline & $20: 50$ & $\begin{array}{l}\text { Began Evaporator pot cool down per Sec } 7.14 \text { of SW9.2-IOP- } \\
\text { EVAP-16H(CC)-1 (Tube Bundle Cooling Water Operations). }\end{array}$ & $\begin{array}{c}28 \mathrm{H} \\
\mathrm{CR}\end{array}$ \\
\hline \multirow[t]{2}{*}{$11 / 24 / 06$} & $03: 55$ & $\begin{array}{l}\text { Due to observation of higher than expected pot press while } \\
\text { agitation with portable through bypass in preparation for caustic } \\
\text { addition, the decision has been made to secure cooling water } \\
\text { flow and portable air compressor and consult engineering for } \\
\text { path forward. }\end{array}$ & $\begin{array}{c}28 \mathrm{H} \\
\mathrm{CR}\end{array}$ \\
\hline & $14: 23$ & $\mathrm{~S} / \mathrm{D}$ portable $\mathrm{A} / \mathrm{C}$ for Maintenance to change filter on lance $\mathrm{GV}$. & $\begin{array}{c}28 \mathrm{H} \\
\mathrm{CR}\end{array}$ \\
\hline \multirow[t]{3}{*}{$11 / 25 / 06$} & 04:39 & Started placing air on lance by portable air compressor. & $\begin{array}{c}28 \mathrm{H} \\
\mathrm{CR}\end{array}$ \\
\hline & $05: 30$ & $\begin{array}{l}\text { Removed air to lance via portable air comp due to not achieving } \\
45 \text { scfm's. Lance reached between } 25-30 \text { scfm's and pot press } \\
\text { reached } 20 \text { "WC. Prior to putting lance on pot level is } 44.1 \text {, } \\
\text { flash tank } \approx 20 \text { ", CRC pump tank } 3.4 \text { and after lance was } \\
\text { removed, pot level } 44.0 \text {, flash tank level 30", and CRC pump } \\
\text { tank is 9.2". }\end{array}$ & $\begin{array}{c}28 \mathrm{H} \\
\mathrm{CR}\end{array}$ \\
\hline & $05: 43$ & Lance GV discharge pressure is still at 4 psi with compressor & $28 \mathrm{H}$ \\
\hline
\end{tabular}




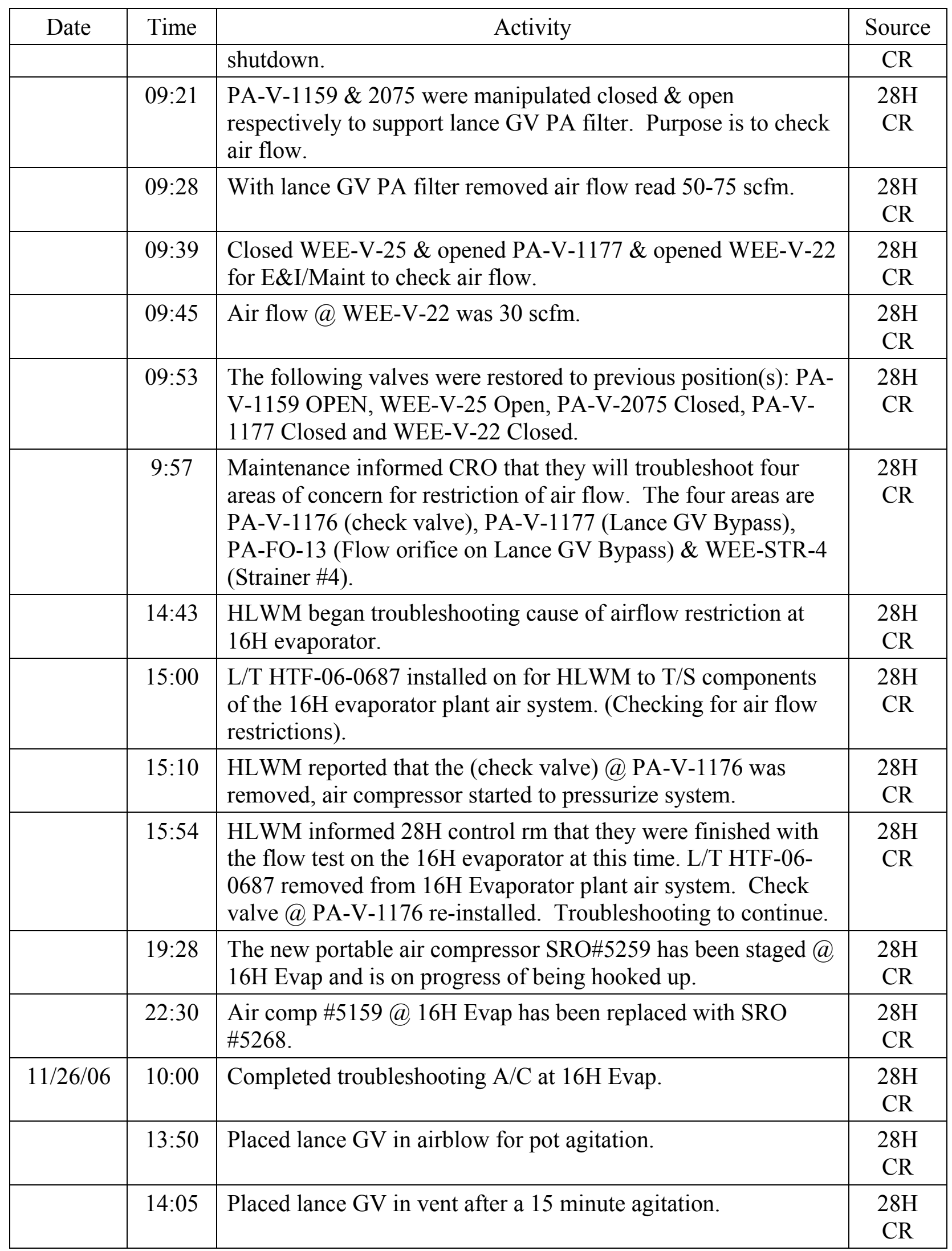




\begin{tabular}{|c|c|c|c|}
\hline Date & Time & Activity & Source \\
\hline & 22:05 & $\begin{array}{l}\text { Installing L/T HTF- } 06-0688 \text { for Maint to remove bypass line @ } \\
\text { lance GV for I\&M to perform inspection to check for } \\
\text { obstructions in lance line. }\end{array}$ & $\begin{array}{c}28 \mathrm{H} \\
\mathrm{CR}\end{array}$ \\
\hline \multirow[t]{3}{*}{$11 / 27 / 06$} & 01:54 & Removing L/T HTF-06-0688 from Lance GV. & $\begin{array}{l}28 \mathrm{H} \\
\mathrm{CR}\end{array}$ \\
\hline & 23:00 & Completed putting air on Lance GV. & $\begin{array}{c}28 \mathrm{H} \\
\mathrm{CR}\end{array}$ \\
\hline & 23:20 & Completed putting water on tube bundle. & $\begin{array}{l}28 \mathrm{H} \\
\mathrm{CR}\end{array}$ \\
\hline \multirow[t]{10}{*}{$11 / 28 / 06$} & 4:59 & Completed empting $1^{\text {st }} 220$ gals of caustic into pot. & $\begin{array}{c}28 \mathrm{H} \\
\mathrm{CR}\end{array}$ \\
\hline & $8: 45$ & Completed adding caustic, added 183 gal. & $\begin{array}{c}28 \mathrm{H} \\
\mathrm{CR}\end{array}$ \\
\hline & $10: 30$ & Started $10 \mathrm{hr}$ agitation cycle. & $\begin{array}{c}28 \mathrm{H} \\
\mathrm{CR}\end{array}$ \\
\hline & $15: 33$ & $\begin{array}{l}\text { Performed shutdown of cooling water to the tube bundle per } \\
7.14 \text {. }\end{array}$ & $\begin{array}{l}28 \mathrm{H} \\
\mathrm{CR}\end{array}$ \\
\hline & 20:30 & Completed $10 \mathrm{hr}$ agitation of pot content. & $\begin{array}{c}28 \mathrm{H} \\
\mathrm{CR}\end{array}$ \\
\hline & $21: 14$ & Began pumping evap pot content to Tk 38. Level 317.63”. & $\begin{array}{c}28 \mathrm{H} \\
\mathrm{CR}\end{array}$ \\
\hline & $21: 52$ & Began adding well water to evaporator pot per sec 4.3 . & $\begin{array}{l}28 \mathrm{H} \\
\mathrm{CR}\end{array}$ \\
\hline & $22: 30$ & Completed adding well water to evaporator pot 63.8 ". & $\begin{array}{c}28 \mathrm{H} \\
\mathrm{CR}\end{array}$ \\
\hline & $22: 34$ & Began pumping evap content to $\mathrm{Tk} 38$. & $\begin{array}{l}28 \mathrm{H} \\
\mathrm{CR}\end{array}$ \\
\hline & 23:02 & Completed pumping content to Tk 38 . level 318.25 ". & $\begin{array}{l}28 \mathrm{H} \\
\mathrm{CR}\end{array}$ \\
\hline \multirow[t]{5}{*}{$11 / 29 / 06$} & $15: 15$ & Began acid addition into evaporator pot @ approx 5 gpm. & $\begin{array}{l}28 \mathrm{H} \\
\mathrm{CR}\end{array}$ \\
\hline & 15:20 & $\begin{array}{l}\text { Increased acid flow to approx } 10 \text { gpm. per sec } 7.12 \text { of Chem } \\
\text { Clean manual. }\end{array}$ & $\begin{array}{c}28 \mathrm{H} \\
\mathrm{CR}\end{array}$ \\
\hline & $16: 36$ & $\begin{array}{l}\text { Unloading of the nitric acid tanker is complete. Evaporator pot } \\
\text { level is } 56.1 \text { " received } 2,073 \text { gallons of nitric into pot. }\end{array}$ & $\begin{array}{l}28 \mathrm{H} \\
\mathrm{CR}\end{array}$ \\
\hline & $17: 23$ & $\begin{array}{l}\text { Completed shutdown of acid addition and nitric acid flush using } \\
\text { approx } 50 \text { gallons of flush water for flush. }\end{array}$ & $\begin{array}{l}28 \mathrm{H} \\
\mathrm{CR}\end{array}$ \\
\hline & 18:00 & $\begin{array}{l}\text { Placed steam on tube bundle per Sec } 4.5 \text { of Evap Chem/Clean } \\
\text { manual. }\end{array}$ & $\begin{array}{l}28 \mathrm{H} \\
\mathrm{CR}\end{array}$ \\
\hline
\end{tabular}


WSRC-STI-2007-00286, REVISON 0

\begin{tabular}{|c|c|c|c|}
\hline Date & Time & Activity & Source \\
\hline & $21: 22$ & $\begin{array}{l}\text { Began pot simmering period } 32 \mathrm{hr} \text { simmering period ends } \\
\text { 12/2/06@ 5:22 hrs. }\end{array}$ & $\begin{array}{c}28 \mathrm{H} \\
\mathrm{CR}\end{array}$ \\
\hline \multirow[t]{4}{*}{$11 / 30 / 06$} & $19: 18$ & $\begin{array}{l}\text { Started adding well water to the pot per sec } 4.3 \text {. This was done } \\
\text { due to a decrease in pot level per sec } 4.4 \text {. }\end{array}$ & $\begin{array}{c}28 \mathrm{H} \\
\mathrm{CR}\end{array}$ \\
\hline & $19: 37$ & $\begin{array}{l}\text { Completed adding well water to the pot per sect } 4.3 \text {. Temp of } \\
\text { pot is } 89.5 \text {. Did not go below } 85^{\circ} \text { low limit. }\end{array}$ & $\begin{array}{c}28 \mathrm{H} \\
\mathrm{CR}\end{array}$ \\
\hline & $20: 41$ & $\begin{array}{l}\text { Started automatic dip tube flush per } 5.1 \text { due to pot level } \\
\text { comparison being }>3 \text { ". }\end{array}$ & $\begin{array}{c}28 \mathrm{H} \\
\mathrm{CR}\end{array}$ \\
\hline & 21:04 & Completed automatic dip tube flush. Used 38 gals FW. & $\begin{array}{c}28 \mathrm{H} \\
\mathrm{CR}\end{array}$ \\
\hline \multirow[t]{5}{*}{$12 / 1 / 06$} & $05: 30$ & $\begin{array}{l}32 \mathrm{hr} \text { simmer of evaporator pot completed. Began subsection } \\
4.4 .3 \text {, shutdown of heating evaporator pot. }\end{array}$ & $\begin{array}{c}28 \mathrm{H} \\
\mathrm{CR}\end{array}$ \\
\hline & $14: 10$ & $\begin{array}{l}\text { Caustic has been added to evaporator cell sump ( } 25 \text { gals). } \\
\text { Agitated for } 30 \text { minutes and sampled. pH results are } 11 .\end{array}$ & $\begin{array}{c}28 \mathrm{H} \\
\mathrm{CR}\end{array}$ \\
\hline & $15: 33$ & $\begin{array}{l}\text { Completed jetting } 323 \text { gals out of evaporator cell sump to Tk } \\
43 \text {. }\end{array}$ & $\begin{array}{c}28 \mathrm{H} \\
\mathrm{CR}\end{array}$ \\
\hline & $16: 11$ & Tube bundle pressurized with water. & $\begin{array}{c}28 \mathrm{H} \\
\mathrm{CR}\end{array}$ \\
\hline & $20: 25$ & Evaporator pot temperature reached $39^{\circ} \mathrm{C}$ at $2000 \mathrm{hrs}$. & $\begin{array}{c}28 \mathrm{H} \\
\mathrm{CR}\end{array}$ \\
\hline $12 / 2 / 06$ & $01: 25$ & $\begin{array}{l}\text { Placed the lance portable air compressor on line; all parameters } \\
\text { are within limits; pot pressure is varying between } 19.1 \text { and 19.6. }\end{array}$ & $\begin{array}{c}28 \mathrm{H} \\
\mathrm{CR}\end{array}$ \\
\hline \multirow[t]{8}{*}{$12 / 2 / 06$} & $01: 30$ & $\begin{array}{l}\text { Had to stop caustic addition procedure to have maintenance } \\
\text { investigate leak discovered when unloading hose was } \\
\text { pressurized with flush water. }\end{array}$ & $\begin{array}{c}28 \mathrm{H} \\
\mathrm{CR}\end{array}$ \\
\hline & $02: 25$ & $\begin{array}{l}\text { Maintenance successfully repaired leaking cam-lok on caustic } \\
\text { tote. }\end{array}$ & $\begin{array}{c}28 \mathrm{H} \\
\mathrm{CR}\end{array}$ \\
\hline & $03: 23$ & $\begin{array}{l}\text { Shutdown pump due to loss of prime to empty tote; estimated } \\
\text { of } 220 \text { gals of caustic added to pot; level is } 59.1^{\prime \prime} \text {; temperature } \\
\text { is } 48^{\circ} \mathrm{C} \text {; R\&HE has arrived to change out totes. }\end{array}$ & $\begin{array}{c}28 \mathrm{H} \\
\mathrm{CR}\end{array}$ \\
\hline & 04:19 & Started caustic pump to empty the second tote. & $\begin{array}{c}28 \mathrm{H} \\
\mathrm{CR}\end{array}$ \\
\hline & 05:01 & $\begin{array}{l}\text { Pump shutdown due to loss of prime; pot level } 64.8^{\prime \prime} \text {; pot } \\
\text { temperature is } 54^{\circ} \mathrm{C} \text {. }\end{array}$ & $\begin{array}{c}28 \mathrm{H} \\
\mathrm{CR}\end{array}$ \\
\hline & $05: 33$ & $\begin{array}{l}\text { Estimated that a total of } 459 \text { gallons of caustic was added to the } \\
\text { evaporator pot. }\end{array}$ & $\begin{array}{c}28 \mathrm{H} \\
\mathrm{CR}\end{array}$ \\
\hline & $06: 20$ & Initiated the 10 hour agitation time. & $\begin{array}{c}28 \mathrm{H} \\
\mathrm{CR}\end{array}$ \\
\hline & $16: 40$ & Completed removing cooling water from tube bundle per & $28 \mathrm{H}$ \\
\hline
\end{tabular}




\begin{tabular}{|c|c|c|c|}
\hline \multirow[t]{10}{*}{ Date } & Time & Activity & Source \\
\hline & & SW9.2-IOP-EVAP-16H(CC)-1 Sec. 7.14 .3 & CR \\
\hline & 17:01 & Started to empty evap pot to Tk 38. & $\begin{array}{c}28 \mathrm{H} \\
\mathrm{CR}\end{array}$ \\
\hline & 17:29 & Completed empting evap pot to Tk 38 . & $\begin{array}{c}28 \mathrm{H} \\
\mathrm{CR}\end{array}$ \\
\hline & $21: 55$ & Started filling the evaporator pot with well water. & $\begin{array}{l}28 \mathrm{H} \\
\mathrm{CR}\end{array}$ \\
\hline & $22: 13$ & $\begin{array}{l}\text { Received OH cell sump HI Level bubbler. Stopped WW } \\
\text { addition until source of leakage can be determined. }\end{array}$ & $\begin{array}{c}28 \mathrm{H} \\
\mathrm{CR}\end{array}$ \\
\hline & $22: 30$ & Sump increase was due to dip tube. & $\begin{array}{l}28 \mathrm{H} \\
\mathrm{CR}\end{array}$ \\
\hline & $22: 34$ & Re-initiated well water addition to evaporator pot. & $\begin{array}{l}28 \mathrm{H} \\
\mathrm{CR}\end{array}$ \\
\hline & $22: 53$ & Completed filling evaporator pot to 95 ”. & $\begin{array}{l}28 \mathrm{H} \\
\mathrm{CR}\end{array}$ \\
\hline & $23: 42$ & Started pumping evaporator pot to Tank 38. Level 319.05 . & $\begin{array}{c}28 \mathrm{H} \\
\mathrm{CR}\end{array}$ \\
\hline \multirow[t]{2}{*}{$12 / 3 / 07$} & 00:27 & $\begin{array}{l}\text { Completed pumping evaporator pot to Tank } 38 \text {. Level } 319.96 \\
3194 \text { gallons FW. }\end{array}$ & $\begin{array}{l}28 \mathrm{H} \\
\mathrm{CR}\end{array}$ \\
\hline & 09:30 & $\begin{array}{l}\text { Completed prejob brief for I\&M to install camera in } 16 \mathrm{H} \\
\text { Evaporator pot to perform inspection. }\end{array}$ & $\begin{array}{c}28 \mathrm{H} \\
\mathrm{CR}\end{array}$ \\
\hline \multirow[t]{4}{*}{$12 / 4 / 07$} & $21: 24$ & Nitric acid pump started. & $\begin{array}{c}28 \mathrm{H} \\
\mathrm{CR}\end{array}$ \\
\hline & $21: 25$ & Flow seen at the funnel; flow at $5.2 \mathrm{gpm}$. & $\begin{array}{c}28 \mathrm{H} \\
\mathrm{CR}\end{array}$ \\
\hline & $21: 37$ & Flow of nitric acid pump at $20 \mathrm{gpm}$. & $\begin{array}{c}28 \mathrm{H} \\
\mathrm{CR}\end{array}$ \\
\hline & 22:58 & $\begin{array}{l}\text { Shutdown nitric acid pump due to levels between } 53 \text { " and } 61 \text { " } \\
\text { (53.1" Blue and 54.2" Red). }\end{array}$ & $\begin{array}{c}28 \mathrm{H} \\
\mathrm{CR}\end{array}$ \\
\hline \multirow[t]{2}{*}{$12 / 5 / 06$} & 00:50 & $\begin{array}{l}\text { Placed steam on tube bundle to initiate heat-up portion of chem } \\
\text { clean section } 4.4 \text {. }\end{array}$ & $\begin{array}{c}28 \mathrm{H} \\
\mathrm{CR}\end{array}$ \\
\hline & 04:10 & $\begin{array}{l}\text { Reached } 85^{\circ} \mathrm{C} \text { in pot; } 32 \text { simmer time initiated; end of } 32 \text { hour } \\
\text { simmer is 12/06/06@ 12:20. }\end{array}$ & $\begin{array}{c}28 \mathrm{H} \\
\mathrm{CR}\end{array}$ \\
\hline \multirow[t]{3}{*}{$12 / 6 / 06$} & 12:11 & Completed heating evaporator pot per section 4.4 . & $\begin{array}{l}28 \mathrm{H} \\
\mathrm{CR}\end{array}$ \\
\hline & $17: 45$ & Completed cooling the evaporator pot. & $\begin{array}{l}28 \mathrm{H} \\
\mathrm{CR}\end{array}$ \\
\hline & $20: 48$ & $\begin{array}{l}\text { Completed installing portable air on the lance GV per SW9.2- } \\
\text { IOP-EVAP-16H(CC)-1 section 7.23. }\end{array}$ & $\begin{array}{l}28 \mathrm{H} \\
\mathrm{CR}\end{array}$ \\
\hline
\end{tabular}


WSRC-STI-2007-00286, REVISON 0

\begin{tabular}{|c|c|c|c|}
\hline Date & Time & Activity & Source \\
\hline & $22: 24$ & Caustic pump started to empty $1^{\text {st }}$ container into evaporator pot. & $\begin{array}{c}28 \mathrm{H} \\
\mathrm{CR}\end{array}$ \\
\hline & $22: 50$ & Completed empting $1^{\text {st }}$ container into evaporator pot. & $\begin{array}{c}28 \mathrm{H} \\
\mathrm{CR}\end{array}$ \\
\hline \multirow[t]{13}{*}{$12 / 7 / 06$} & 01:04 & Caustic pump started to empty $2^{\text {nd }}$ container into evaporator pot. & $\begin{array}{c}28 \mathrm{H} \\
\mathrm{CR}\end{array}$ \\
\hline & $01: 23$ & $\begin{array}{l}\text { Completed emptying } 2^{\text {nd }} \text { container of caustic into evaporator } \\
\text { pot. } 10 \text { hour simmer time has begun. }\end{array}$ & $\begin{array}{c}28 \mathrm{H} \\
\mathrm{CR}\end{array}$ \\
\hline & $9: 03$ & Secured cooling water to tube bundle. & $\begin{array}{c}28 \mathrm{H} \\
\mathrm{CR}\end{array}$ \\
\hline & $11: 26$ & Completed 10 hrs simmer of evaporator pot. & $\begin{array}{c}28 \mathrm{H} \\
\mathrm{CR}\end{array}$ \\
\hline & $11: 59$ & $\begin{array}{l}\text { Begin pumping evaporator pot to Tk } 38 \text { level } 319.86 \text { " pot level } \\
\text { at } 64.0 \text { ". }\end{array}$ & $\begin{array}{c}28 \mathrm{H} \\
\mathrm{CR}\end{array}$ \\
\hline & $12: 22$ & Completed pumping evaporator pot to Tk 38. & $\begin{array}{c}28 \mathrm{H} \\
\mathrm{CR}\end{array}$ \\
\hline & $12: 26$ & Began filling pot with well water. & $\begin{array}{c}28 \mathrm{H} \\
\mathrm{CR}\end{array}$ \\
\hline & 13:00 & Completed filling evaporator pot with well water. & $\begin{array}{c}28 \mathrm{H} \\
\mathrm{CR}\end{array}$ \\
\hline & $13: 03$ & Began pumping evaporator pot to Tk 38 level 320.42”. & $\begin{array}{c}28 \mathrm{H} \\
\mathrm{CR}\end{array}$ \\
\hline & $13: 45$ & $\begin{array}{l}\text { Completed pump evaporator to } \mathrm{Tk} 38 \text {, also performed } \\
\text { shutdown per sec } 4.6 \text {. }\end{array}$ & $\begin{array}{c}28 \mathrm{H} \\
\mathrm{CR}\end{array}$ \\
\hline & $20: 21$ & Started adding nitric acid to evaporator pot. & $\begin{array}{c}28 \mathrm{H} \\
\mathrm{CR}\end{array}$ \\
\hline & $21: 45$ & $\begin{array}{l}\text { Completed adding nitric acid to evaporator pot. Added } 2060 \\
\text { gallons. }\end{array}$ & $\begin{array}{c}28 \mathrm{H} \\
\mathrm{CR}\end{array}$ \\
\hline & $23: 28$ & Placed steam on tube bundle pre section 4.4 . & $\begin{array}{c}28 \mathrm{H} \\
\mathrm{CR}\end{array}$ \\
\hline \multirow[t]{4}{*}{$12 / 8 / 06$} & $02: 38$ & Reached $85^{\circ} \mathrm{C}$. & $\begin{array}{c}28 \mathrm{H} \\
\mathrm{CR}\end{array}$ \\
\hline & $02: 58$ & Removed steam from the tube bundle. & $\begin{array}{l}28 \mathrm{H} \\
\mathrm{CR}\end{array}$ \\
\hline & $05: 35$ & E\&I found bad breaker for AIV. & $\begin{array}{c}28 \mathrm{H} \\
\mathrm{CR}\end{array}$ \\
\hline & 08:58 & $\begin{array}{l}\text { E\&I completed changing breaker that feed the AIV. Breaker } \\
\text { was determined to be faulty by E\&I on previous shift when } \\
\text { AIV inadvertently closed during pot heatup. }\end{array}$ & $\begin{array}{c}28 \mathrm{H} \\
\mathrm{CR}\end{array}$ \\
\hline
\end{tabular}




\begin{tabular}{|c|c|c|c|}
\hline Date & Time & Activity & Source \\
\hline \multirow[t]{5}{*}{$12 / 09 / 06$} & 07:35 & Entered AOP-009 for loss of steam. & $\begin{array}{c}28 \mathrm{H} \\
\mathrm{CR}\end{array}$ \\
\hline & 07:45 & $\begin{array}{l}\text { SUD reported that a transmitter had frozen causing steam } \\
\text { supply valve to close. Steam being supplied manually by SUD. }\end{array}$ & $\begin{array}{c}28 \mathrm{H} \\
\mathrm{CR}\end{array}$ \\
\hline & $13: 32$ & $\begin{array}{l}\text { Completed establishing cooling water flow through tube } \\
\text { bundle. }\end{array}$ & $\begin{array}{l}28 \mathrm{H} \\
\mathrm{CR}\end{array}$ \\
\hline & 18:07 & Reached $39^{\circ}$ on pot temperature. & $\begin{array}{l}28 \mathrm{H} \\
\mathrm{CR}\end{array}$ \\
\hline & $21: 52$ & Started adding caustic. & $\begin{array}{c}28 \mathrm{H} \\
\mathrm{CR}\end{array}$ \\
\hline \multirow[t]{9}{*}{$12 / 10 / 06$} & 00:48 & Completed caustic addition to the pot, 434 gallons. & $\begin{array}{l}28 \mathrm{H} \\
\mathrm{CR}\end{array}$ \\
\hline & $01: 20$ & $\begin{array}{l}\text { Completed flushing of the caustic line the } 10 \text { hour agitation } \\
\text { starts now and will be up at 11:20 today. }\end{array}$ & $\begin{array}{c}28 \mathrm{H} \\
\mathrm{CR}\end{array}$ \\
\hline & $11: 57$ & Cooling water has been secured to the tube bundle. & $\begin{array}{c}28 \mathrm{H} \\
\mathrm{CR}\end{array}$ \\
\hline & 12:18 & Started pumping evaporator pot to Tk 38 per section 4.6. & $\begin{array}{l}28 \mathrm{H} \\
\mathrm{CR}\end{array}$ \\
\hline & $12: 53$ & Completed emptying evaporator pot per section 4.6. & $\begin{array}{c}28 \mathrm{H} \\
\mathrm{CR}\end{array}$ \\
\hline & $13: 12$ & Started filling evaporator pot with well water per section 4.3 . & $\begin{array}{c}28 \mathrm{H} \\
\mathrm{CR}\end{array}$ \\
\hline & $13: 45$ & $\begin{array}{l}\text { Completed filling evaporator pot with well water per section } \\
4.3 \text {. }\end{array}$ & $\begin{array}{c}28 \mathrm{H} \\
\mathrm{CR}\end{array}$ \\
\hline & 13:52 & Started pumping evaporator pot per section 4.6. & $\begin{array}{c}28 \mathrm{H} \\
\mathrm{CR}\end{array}$ \\
\hline & $14: 33$ & Completed pumping evaporator pot to Tk38 per section 4.6 . & $\begin{array}{c}28 \mathrm{H} \\
\mathrm{CR}\end{array}$ \\
\hline \multirow[t]{6}{*}{$12 / 11 / 06$} & $12: 35$ & $\begin{array}{l}\text { Completed camera inspection on } 16 \mathrm{H} \text { evaporator and riser plug } \\
\text { has been installed. }\end{array}$ & $\begin{array}{c}28 \mathrm{H} \\
\mathrm{CR}\end{array}$ \\
\hline & 16:08 & Started adding first tote of caustic to pot. & $\begin{array}{c}28 \mathrm{H} \\
\mathrm{CR}\end{array}$ \\
\hline & $16: 38$ & Completed adding first tote of caustic. & $\begin{array}{c}28 \mathrm{H} \\
\mathrm{CR}\end{array}$ \\
\hline & $17: 36$ & Started adding second tote of caustic. & $\begin{array}{c}28 \mathrm{H} \\
\mathrm{CR}\end{array}$ \\
\hline & 18:17 & Completed adding 521 gals of caustic to evaporator pot. & $\begin{array}{c}28 \mathrm{H} \\
\mathrm{CR}\end{array}$ \\
\hline & 19:43 & Completed demister flush, used 337 gallons flush water. & $28 \mathrm{H}$ \\
\hline
\end{tabular}


WSRC-STI-2007-00286, REVISON 0

\begin{tabular}{|c|c|c|c|}
\hline Date & Time & Activity & Source \\
\hline & & & $\mathrm{CR}$ \\
\hline & $20: 07$ & Completed flushing the evaporator dip tube, used 28.0 gallons. & $\begin{array}{c}28 \mathrm{H} \\
\mathrm{CR}\end{array}$ \\
\hline & $20: 35$ & $\begin{array}{l}\text { Completed filling the evaporator pot, final level is } 61.5 \text { inches. } \\
\text { Added approx } 1642 \text { gallons. }\end{array}$ & $\begin{array}{c}28 \mathrm{H} \\
\mathrm{CR}\end{array}$ \\
\hline & $20: 44$ & $\begin{array}{l}\text { Started evaporator pot heat up per SW9.2-IOP-EVAP- } \\
16 \mathrm{H}(\mathrm{CC})-1 \text {, section } 5.4 \text { Starting pot temperature } 40^{\circ} \mathrm{C} \text {. }\end{array}$ & $\begin{array}{c}28 \mathrm{H} \\
\mathrm{CR}\end{array}$ \\
\hline \multirow[t]{5}{*}{$12 / 12 / 06$} & $00: 30$ & Evaporator pot reached temperature of $100^{\circ} \mathrm{C}$ & $\begin{array}{c}28 \mathrm{H} \\
\mathrm{CR}\end{array}$ \\
\hline & $04: 30$ & Shutdown steam to the evaporator tube bundle. & $\begin{array}{c}28 \mathrm{H} \\
\mathrm{CR}\end{array}$ \\
\hline & 05:02 & $\begin{array}{l}\text { Completed operation of evaporator cell sprays per SW9.2-IOP- } \\
\text { EVAP-16H, section } 8.8 \text {. Added } 196 \text { gallons flush water to cell } \\
\text { sump. }\end{array}$ & $\begin{array}{c}28 \mathrm{H} \\
\mathrm{CR}\end{array}$ \\
\hline & $10: 05$ & Completed adding 35 gallons of caustic to evaporator cell. & $\begin{array}{c}28 \mathrm{H} \\
\mathrm{CR}\end{array}$ \\
\hline & $13: 22$ & $\begin{array}{l}\text { Completed jetting evaporator cell sump to Tank } 43 \text {. Jetted } 444 \\
\text { gallons. }\end{array}$ & $\begin{array}{c}28 \mathrm{H} \\
\mathrm{CR}\end{array}$ \\
\hline $12 / 20 / 06$ & $22: 14$ & $\begin{array}{l}\text { Steam on to tube bundle. Normal evaporator operation } \\
\text { resumed. }\end{array}$ & $\begin{array}{c}28 \mathrm{H} \\
\mathrm{CR}\end{array}$ \\
\hline
\end{tabular}




\section{APPENDIX B. LESSONS LEARNED DURING OUTAGE}

The Chemical Cleaning of the 242-16H (2H) Evaporator using nitric acid in December 2006 was the third pot cleaning. The second chemical cleaning used pure $19 \mathrm{M}$ sodium hydroxide $(\mathrm{NaOH})$. This method was tried since the impact on the Documented Safety Basis (DSA) was minimal. The lesson learned from the use of $\mathrm{NaOH}$ was that although the scale dissolved, the rate was not fast enough to support the H Tank Farm (HTF) mission.

The differences between the acid cleaning evolutions are as follows:

\begin{tabular}{|l|l|}
\hline \multicolumn{1}{|c|}{ May 2001 } & \multicolumn{1}{c|}{ December 2006 } \\
\hline $\begin{array}{l}\text { Used 1.5M nitric acid containing depleted } \\
\text { Uranyl Nitrate. }\end{array}$ & Used commercial grade 1.5M nitric acid. \\
\hline $\begin{array}{l}\text { The pot was cleaned to a true level of } \\
\text { approximately 95 inches. }\end{array}$ & $\begin{array}{l}\text { The pot was cleaned to a true level of } \\
\text { approximately 55 inches. }\end{array}$ \\
\hline $\begin{array}{l}\text { Required the used of a 5,000 gallon } \\
\text { neutralization tank. }\end{array}$ & Neutralization was performed in the pot. \\
\hline $\begin{array}{l}\text { Neutralized waste was transferred to Tank 42. } \\
\text { Neutralized waste was discharged directly into } \\
\text { the tank. }\end{array}$ & $\begin{array}{l}\text { Neutralized waste was transferred to Tank } \\
38 .\end{array}$ \\
\hline
\end{tabular}

To minimize cost and schedule, the May 2001 system design, equipment and arrangements were used. The same lift jumper was used except it was redesigned to connect to the wall nozzle to Tank 38 instead of Tank 42. In addition a second isolation valve was added to support a DSA requirement for double valve isolation when acid was in the pot. The only problem encountered during operation of the lift jumper is that it initially failed to pump waste from the pot. After much troubleshooting it was determined that there was blockage in the east lift line. The evaporator cell covers and the lift jumper had to be removed, and the lift line pressure washed to remove the plug. Once the line was cleaned, no additional problems were encountered.

It was determined that evaporator cleaning would be required on a periodic basis and that the acid/caustic cleaning system design should be revised to support repeated use. Whereas the 2001 system was considered to be a one time use only, system arrangement and extended operational life was not a consideration.

The caustic addition flow rate was required to be low to control the exothermic reaction between the acid and caustic. During testing a concern was raised that the pump head was not sufficient to deliver the caustic to the funnel because in 2001 the caustic was pumped to a temporary neutralization tank. Although the pump head was sufficient to handle the elevation difference, 
the total line losses were not known. To ensure that there was adequate suction and discharge head, the caustic pump was replaced with a higher capacity pump.

It was also learned during shop testing that the flow rate of water was not as expected considering the capacity of the pump. The original design required the use of cam lock connections without a check valve. Engineering decided to use Hansen quick disconnect fittings to reduce the possibility of acid and caustic leaks during hose disconnection. After the check valves in the Hansen connectors were removed, the flow was as expected. Additionally, since operational life of the original system was not a concern, the chemical compatibility of its gaskets was not evaluated. The increase in operational life identified various gaskets and seals which were not compatible for long term contact with either the acid or the caustic. It was discovered in the testing phase that the Hansen connector seal components contained some of these incompatible materials which required removal and replacement before the system could be placed in service. Vendor drawing did not show these O-rings nor did it provide a part number for the required O-rings. The need for EPDM gaskets for the Hansen disconnects was verbally communicated to Work Control. Work Control relayed the information to Procurement. Teflon gaskets were received instead and the EPDM gaskets had to be reordered. It is possible that the right gaskets were ordered but the wrong gaskets were sent by the vendor.

Although the flexible hoses did not leak during the evaporator cleaning operations, problems were encountered by the supplier. The flexible hose supplier claimed that the hoses were hydrostatically test and passed. But when Maintenance performed a verification hydrostatic test as part of the assembled system post modification test many of them leaked. Upon investigation it was learned that the first vendor did not have the capability of crimping the hose as required. Therefore, the work was subcontracted and the subcontractor did not hydrostatically test the hoses properly. In the future, when there is a requirement for adding hose ends and hydrostatic testing is required, the hoses will be ordered from a vendor who is capable of performing both tasks. This will ensure receipt of a quality product.

When the chemical addition skid was first positioned at the evaporator and the hoses connected, the hoses were to short. After rotating the skid $180^{\circ}$, the hoses were too long. Again, disciplined reviews by Engineering, Operations, and Maintenance were not adequate and caused rework.

Another Engineering lesson learned was associated with the air flow to the evaporator lance for mixing the acid and the caustic. The same flow meter was specified as used in May 2001. However, when air was applied, the desired mixing flow rate was not achieved. After much field testing it was finally realized that the flow meter was not calibrated for the pressure and temperature at which it was being operated. After the formula was obtained from the vendor to determine a correction factor, the equipment performed satisfactorily.

Since much of the process used for the December 2006 was the same as used in May 2001, many of the same procedures were used. The primary changes to the procedures were the result of the 
different DSA requirements and not using a neutralization tank. This led some operations personnel to believe that the water runs using the procedures were not required. As one can see by reviewing the Shift Manager's and control room operator's log books, water runs should have been conducted. Fortunately, only a small acid spill occurred during the cleaning activities.

In general, the problems encountered during the 2006 chemical cleaning of the $2 \mathrm{H}$ Evaporator pot were minimal in part due to reviewing lessons learned from 2001. The primary lesson learned from this cleaning effort is "Do not assume that what worked last time will work next time unless disciplined reviews by Engineering, Operations, and Maintenance are managed consistent with desired priorities." 


\section{APPENDIX C. 2H POT MEASUREMENTS}

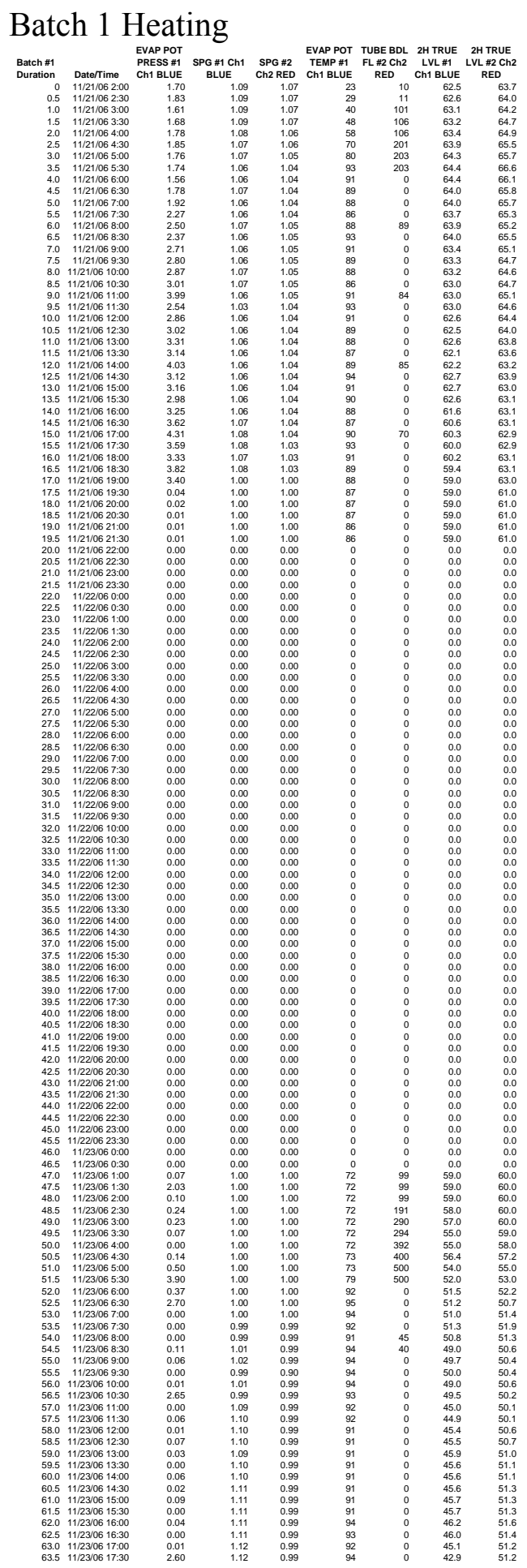

Page 29 of 37 
WSRC-STI-2007-00286, REVISON 0

\section{Batch 2 Heating}

\begin{tabular}{|c|c|c|c|c|c|c|c|c|}
\hline $\begin{array}{l}\text { Batch \#2 } \\
\text { Duration }\end{array}$ & Date/Time & $\begin{array}{l}\text { PRESS \#1 } \\
\text { Ch1 BLUE }\end{array}$ & $\begin{array}{c}\text { SPG \#1 } \\
\text { Ch1 BLUE }\end{array}$ & $\begin{array}{c}\text { SPG \#2 } \\
\text { Ch2 RED }\end{array}$ & $\begin{array}{l}\text { TEMP \#1 } \\
\text { Ch1 BLUE }\end{array}$ & $\begin{array}{l}\mathrm{FL} \# 2 \mathrm{Ch} 2 \\
\text { RED }\end{array}$ & $\begin{array}{c}\text { LVL \#1 } \\
\text { Ch1 BLUE }\end{array}$ & $\begin{array}{c}\text { LVL \#2 } \\
\text { Ch2 RED }\end{array}$ \\
\hline 0 & $11 / 29 / 06$ 18:00 & 1.92 & 1.00 & 1.00 & 24 & 100 & 57.7 & 59.0 \\
\hline 0.0 & $11 / 29 / 0618: 30$ & 1.90 & 1.06 & 1.04 & 35 & 100 & 58.0 & 59.3 \\
\hline 0.0 & $11 / 29 / 06$ 19:00 & 1.80 & 1.06 & 1.04 & 42 & 165 & 28.1 & 59.5 \\
\hline 0.0 & 11/29/06 19:30 & 2.02 & 1.06 & 1.04 & 51 & 192 & 58.4 & 59.8 \\
\hline 0.0 & $11 / 29 / 0620: 00$ & 2.08 & 1.05 & 1.03 & 62 & 191 & 58.7 & 60.1 \\
\hline 0.0 & 11/29/06 20:30 & 2.17 & 1.04 & 1.03 & 73 & 235 & 59.3 & 60.6 \\
\hline 0.0 & 11/29/06 21:00 & 2.21 & 1.03 & 1.02 & 84 & 236 & 59.7 & 61.1 \\
\hline 0.0 & $11 / 29 / 0621: 30$ & 2.05 & 1.06 & 1.02 & 92 & 0 & 59.8 & 61.4 \\
\hline 0.5 & $11 / 29 / 0622: 00$ & 1.62 & 1.03 & 1.02 & 90 & 0 & 59.6 & 61.0 \\
\hline 1.0 & $11 / 29 / 0622: 30$ & 1.75 & 1.04 & 1.02 & 88 & 0 & 59.2 & 60.7 \\
\hline 1.5 & $11 / 29 / 0623: 00$ & 2.69 & 1.03 & 1.02 & 92 & 0 & 59.2 & 60.9 \\
\hline 2.0 & $11 / 29 / 0623: 30$ & 2.00 & 1.03 & 1.02 & 90 & 0 & 59.2 & 60.7 \\
\hline 2.5 & 11/30/06 0:00 & 2.20 & 1.04 & 1.02 & 89 & 0 & 59.1 & 60.3 \\
\hline 3.0 & $11 / 30 / 060: 30$ & 2.70 & 1.03 & 1.01 & 93 & 0 & 59.1 & 60.7 \\
\hline 3.5 & $11 / 30 / 061: 00$ & 2.60 & 1.03 & 1.02 & 93 & 0 & 58.7 & 30.2 \\
\hline 4.0 & 11/30/06 1:30 & 2.60 & 1.03 & 1.02 & 92 & 0 & 58.5 & 59.9 \\
\hline 4.5 & $11 / 30 / 062: 00$ & 2.70 & 1.04 & 1.02 & 89 & 0 & 58.1 & 59.5 \\
\hline 5.0 & $11 / 30 / 062: 30$ & 3.10 & 1.04 & 1.02 & 92 & 75 & 58.1 & 59.4 \\
\hline 5.5 & $11 / 30 / 063: 00$ & 2.70 & 1.04 & 1.02 & 91 & 0 & 60.0 & 60.8 \\
\hline 6.0 & 11/30/06 3:30 & 3.00 & 1.04 & 1.02 & 89 & 0 & 57.5 & 58.7 \\
\hline 6.5 & $11 / 30 / 064: 00$ & 2.70 & 1.04 & 1.02 & 88 & 75 & 57.5 & 58.8 \\
\hline 7.0 & $11 / 30 / 064: 30$ & 3.70 & 1.03 & 1.02 & 93 & 0 & 57.6 & 59.0 \\
\hline 7.5 & $11 / 30 / 065: 00$ & 3.50 & 1.03 & 1.01 & 93 & 0 & 57.2 & 58.6 \\
\hline 8.0 & $11 / 30 / 065: 30$ & 3.00 & 1.04 & 1.02 & 93 & 0 & 56.9 & 58.3 \\
\hline 8.5 & $11 / 30 / 066: 00$ & 3.00 & 1.04 & 1.02 & 91 & 0 & 56.6 & 57.9 \\
\hline 9.0 & 11/30/06 6:30 & 3.49 & 1.04 & 1.03 & 89 & 0 & 56.3 & 57.7 \\
\hline 9.5 & $11 / 30 / 067: 00$ & 3.10 & 1.04 & 1.03 & 88 & 0 & 56.2 & 57.4 \\
\hline 10.0 & $11 / 30 / 067: 30$ & 3.64 & 1.04 & 1.03 & 87 & 0 & 55.9 & 57.3 \\
\hline 10.5 & $11 / 30 / 068: 00$ & 4.00 & 1.04 & 1.03 & 91 & 100 & 56.0 & 57.5 \\
\hline 11.0 & $11 / 30 / 068: 30$ & 3.37 & 1.04 & 1.02 & 90 & 0 & 56.0 & 57.2 \\
\hline 11.5 & $11 / 30 / 069: 00$ & 3.39 & 1.04 & 1.02 & 88 & 0 & 55.0 & 57.0 \\
\hline 12.0 & 11/30/06 9:30 & 3.42 & 1.04 & 1.01 & 92 & 0 & 55.8 & 57.2 \\
\hline 12.5 & $11 / 30 / 0610: 00$ & 3.65 & 1.04 & 1.02 & 90 & 0 & 55.2 & 56.8 \\
\hline 13.0 & $11 / 30 / 0610: 30$ & 3.30 & 1.00 & 1.00 & 89 & 0 & 55.0 & 56.0 \\
\hline 13.5 & 11/30/06 11:00 & 3.20 & 1.00 & 1.00 & 88 & 0 & 55.0 & 56.0 \\
\hline 14.0 & 11/30/06 11:30 & 3.90 & 1.00 & 1.00 & 88 & 0 & 55.0 & 56.0 \\
\hline 14.5 & $11 / 30 / 0612: 00$ & 3.70 & 1.00 & 1.00 & 94 & 0 & 55.0 & 57.0 \\
\hline 15.0 & 11/30/06 12:30 & 3.70 & 1.00 & 1.00 & 92 & 0 & 55.0 & 57.0 \\
\hline 15.5 & $11 / 30 / 06$ 13:00 & 3.80 & 1.00 & 1.00 & 90 & 0 & 55.0 & 57.0 \\
\hline 16.0 & $11 / 30 / 06$ 13:30 & 4.09 & 1.00 & 1.00 & 88 & 0 & 54.9 & 56.0 \\
\hline 16.5 & 11/30/06 14:00 & 3.60 & 1.00 & 1.00 & 87 & 0 & 55.0 & 56.0 \\
\hline 17.0 & $11 / 30 / 0614: 30$ & 3.30 & 1.00 & 1.00 & 93 & 0 & 56.0 & 57.0 \\
\hline 17.5 & $11 / 30 / 06$ 15:00 & 4.00 & 1.00 & 1.00 & 92 & 0 & 54.0 & 57.0 \\
\hline 18.0 & $11 / 30 / 0615: 30$ & 3.70 & 1.00 & 1.00 & 92 & 0 & 54.0 & 56.0 \\
\hline 18.5 & $11 / 30 / 06$ 16:00 & 3.90 & 1.00 & 1.00 & 92 & 0 & 54.0 & 56.0 \\
\hline 19.0 & $11 / 30 / 06$ 16:30 & 3.90 & 1.00 & 1.00 & 93 & 0 & 53.0 & 55.0 \\
\hline 19.5 & $11 / 30 / 0617: 00$ & 3.35 & 1.00 & 1.00 & 93 & 0 & 53.0 & 56.0 \\
\hline 20.0 & $11 / 30 / 06$ 17:30 & 4.32 & 1.00 & 1.00 & 93 & 0 & 53.0 & 55.0 \\
\hline 20.5 & $11 / 30 / 0618: 00$ & 0.00 & 1.00 & 1.00 & 93 & 0 & 52.0 & 54.0 \\
\hline 21.0 & $11 / 30 / 06$ 18:30 & 4.20 & 1.00 & 1.00 & 94 & 0 & 52.6 & 55.0 \\
\hline 21.5 & $11 / 30 / 06$ 19:00 & 4.30 & 1.00 & 1.00 & 93 & 0 & 52.2 & 55.1 \\
\hline 22.0 & $11 / 30 / 06$ 19:30 & 5.20 & 1.02 & 1.00 & 91 & 93 & 58.1 & 60.5 \\
\hline 22.5 & $11 / 30 / 0620: 00$ & 4.00 & 1.01 & 1.00 & 91 & 0 & 59.5 & 60.4 \\
\hline 23.0 & $11 / 30 / 0620: 30$ & 4.10 & 1.03 & 1.00 & 90 & 0 & 57.5 & 60.2 \\
\hline 23.5 & $11 / 30 / 06$ 21:00 & 2.00 & 1.05 & 1.02 & 87 & 0 & 57.7 & 59.3 \\
\hline 24.0 & $11 / 30 / 0621: 30$ & 2.30 & 1.04 & 1.02 & 92 & 75 & 57.7 & 59.6 \\
\hline 24.5 & $11 / 30 / 0622: 00$ & 2.00 & 1.04 & 1.02 & 92 & 0 & 57.4 & 59.2 \\
\hline 25.0 & $11 / 30 / 0622: 30$ & 1.90 & 1.04 & 1.02 & 90 & 0 & 57.3 & 59.0 \\
\hline 25.5 & $11 / 30 / 0623: 00$ & 2.70 & 1.04 & 1.02 & 93 & 0 & 57.0 & 59.0 \\
\hline 26.0 & $11 / 30 / 0623: 30$ & 2.60 & 1.04 & 1.02 & 93 & 0 & 56.7 & 58.7 \\
\hline 26.5 & $12 / 1 / 060: 00$ & 2.10 & 1.05 & 1.01 & 93 & 0 & 56.7 & 58.7 \\
\hline 27.0 & $12 / 1 / 060: 30$ & 2.90 & 1.05 & 1.01 & 91 & 0 & 56.2 & 58.2 \\
\hline 27.5 & $12 / 1 / 061: 00$ & 2.40 & 1.05 & 1.02 & 90 & 0 & 56.2 & 58.2 \\
\hline 28.0 & $12 / 1 / 061: 30$ & 2.40 & 1.05 & 1.01 & 93 & 0 & 56.0 & 58.1 \\
\hline 28.5 & $12 / 1 / 062: 00$ & 2.40 & 1.05 & 1.02 & 91 & 0 & 55.7 & 56.6 \\
\hline 29.0 & $12 / 1 / 062: 30$ & 2.70 & 1.05 & 1.02 & 89 & 0 & 55.4 & 57.2 \\
\hline 29.5 & $12 / 1 / 063: 00$ & 3.00 & 1.05 & 1.02 & 93 & 0 & 55.5 & 57.5 \\
\hline 30.0 & $12 / 1 / 063: 30$ & 2.90 & 1.05 & 1.02 & 93 & 0 & 55.5 & 57.6 \\
\hline 30.5 & $12 / 1 / 064: 00$ & 2.50 & 1.04 & 1.01 & 94 & 0 & 54.7 & 56.7 \\
\hline 31.0 & $12 / 1 / 064: 30$ & 2.50 & 1.05 & 1.02 & 91 & 0 & 54.1 & 56.0 \\
\hline 31.5 & $12 / 1 / 065: 00$ & 2.80 & 1.05 & 1.02 & 90 & 0 & 54.0 & 55.9 \\
\hline 32.0 & $12 / 1 / 065: 30$ & 2.70 & 1.05 & 1.02 & 87 & 0 & 53.7 & 55.5 \\
\hline
\end{tabular}

Page 30 of 37 
WSRC-STI-2007-00286, REVISON 0

Batch 3 Heating

\begin{tabular}{|c|c|c|c|c|c|c|c|c|}
\hline $\begin{array}{l}\text { Batch \#3 } \\
\text { Duration }\end{array}$ & Date/Time & $\begin{array}{l}\text { EVAP POT } \\
\text { PRESS \#1 } \\
\text { Ch1 BLUE }\end{array}$ & $\begin{array}{c}\text { SPG \#1 } \\
\text { Ch1 BLUE }\end{array}$ & $\begin{array}{c}\text { SPG \#2 } \\
\text { Ch2 RED }\end{array}$ & $\begin{array}{l}\text { EVAP POT } \\
\text { TEMP \#1 } \\
\text { Ch1 BLUE }\end{array}$ & $\begin{array}{l}\text { TUBE BDL } \\
\text { FL \#2 Ch2 } \\
\text { RED }\end{array}$ & $\begin{array}{l}\text { 2H TRUE } \\
\text { LVL \#1 Ch1 } \\
\text { BLUE }\end{array}$ & $\begin{array}{l}\text { 2H TRUE } \\
\text { LVL \#2 } \\
\text { Ch2 RED }\end{array}$ \\
\hline 0.5 & $12 / 5 / 061: 00$ & 2.10 & 1.06 & 1.04 & 19 & 10 & 54.6 & 55.9 \\
\hline 1.0 & $12 / 5 / 061: 30$ & 2.20 & 1.06 & 1.04 & 27 & 11 & 54.6 & 56.1 \\
\hline 1.5 & $12 / 5 / 06$ 2:00 & 2.00 & 1.05 & 1.04 & 37 & 101 & 55.1 & 56.3 \\
\hline 2.0 & $12 / 5 / 062: 30$ & 1.9 & 1.05 & 1.04 & 47 & 39 & 55.4 & 56.7 \\
\hline 2.5 & 12/5/06 3:00 & 2.10 & 1.05 & 1.04 & 57 & 47 & 55.5 & 56.5 \\
\hline 3.0 & $12 / 5 / 063: 30$ & 2.30 & 1.04 & 1.03 & 69 & 85 & 55.9 & 57.3 \\
\hline 3.5 & $12 / 5 / 064: 00$ & 2.50 & 1.03 & 1.02 & 81 & 90 & 56.9 & 57.8 \\
\hline 4.0 & $12 / 5 / 064: 30$ & 3.01 & 1.02 & 1.00 & 92 & 160 & 56.9 & 58.4 \\
\hline 4.5 & $12 / 5 / 06$ 5:00 & 2.17 & 1.03 & 1.00 & 93 & 195 & 56.5 & 58.3 \\
\hline 5.0 & $12 / 5 / 065: 30$ & 2.80 & 1.02 & 1.00 & 90 & 225 & 56.1 & 57.9 \\
\hline 5.5 & $12 / 5 / 06$ 6:00 & 2.80 & 1.03 & 1.00 & 89 & 243 & 56.1 & 57.6 \\
\hline 6.0 & $12 / 5 / 066: 30$ & 3.70 & 1.03 & 1.01 & 90 & 0 & 55.7 & 57.6 \\
\hline 6.5 & 12/5/06 7:00 & 3.50 & 1.03 & 1.01 & 90 & 0 & 55.8 & 57.3 \\
\hline 7.0 & $12 / 5 / 067: 30$ & 3.60 & 1.03 & 1.01 & 88 & 0 & 55.5 & 57.1 \\
\hline 7.5 & 12/5/06 8:00 & 4.00 & 1.03 & 1.00 & 90 & 0 & 55.5 & 57.0 \\
\hline 8.0 & $12 / 5 / 068: 30$ & 3.50 & 1.03 & 1.01 & 89 & 0 & 55.3 & 56.9 \\
\hline 8.5 & 12/5/06 9:00 & 3.80 & 1.03 & 1.01 & 88 & 0 & 55.0 & 56.7 \\
\hline 9.0 & $12 / 5 / 069: 30$ & 4.50 & 1.03 & 1.01 & 92 & 0 & 55.0 & 56.8 \\
\hline 9.5 & $12 / 5 / 0610: 00$ & 3.50 & 1.03 & 1.01 & 90 & 0 & 54.9 & 56.6 \\
\hline 10.0 & 12/5/06 10:30 & 3.30 & 1.03 & 1.01 & 89 & 0 & 54.8 & 56.1 \\
\hline 10.5 & 12/5/06 11:00 & 3.50 & 1.03 & 1.01 & 87 & 30 & 54.3 & 56.2 \\
\hline 11.0 & 12/5/06 11:30 & 3.60 & 1.03 & 1.01 & 91 & 0 & 54.3 & 56.1 \\
\hline 11.5 & $12 / 5 / 0612: 00$ & 3.40 & 1.03 & 1.01 & 89 & 0 & 54.2 & 55.8 \\
\hline 12.0 & $12 / 5 / 0612: 30$ & 4.10 & 1.03 & 1.01 & 87 & 0 & 54.1 & 55.4 \\
\hline 12.5 & $12 / 5 / 0613: 00$ & 4.50 & 1.03 & 1.01 & 91 & 0 & 53.9 & 55.7 \\
\hline 13.0 & $12 / 5 / 0613: 30$ & 4.30 & 1.03 & 1.01 & 93 & 0 & 53.9 & 55.8 \\
\hline 13.5 & $12 / 5 / 0614: 00$ & 3.70 & 1.03 & 1.01 & 90 & 0 & 53.4 & 55.2 \\
\hline 14.0 & 12/5/06 14:30 & 4.00 & 1.03 & 1.01 & 89 & 0 & 53.1 & 55.0 \\
\hline 14.5 & $12 / 5 / 0615: 00$ & 3.60 & 1.03 & 1.01 & 87 & 0 & 53.1 & 54.7 \\
\hline 15.0 & $12 / 5 / 0615: 30$ & 4.30 & 1.03 & 1.01 & 86 & 23 & 53.1 & 54.7 \\
\hline 15.5 & $12 / 5 / 0616: 00$ & 4.50 & 1.03 & 1.01 & 90 & 21 & 52.9 & 54.7 \\
\hline 16.0 & $12 / 5 / 0616: 30$ & 4.00 & 1.03 & 1.01 & 92 & 0 & 53.1 & 54.7 \\
\hline 16.5 & $12 / 5 / 0617: 00$ & 3.60 & 1.03 & 1.01 & 90 & 0 & 52.8 & 54.2 \\
\hline 17.0 & $12 / 5 / 0617: 30$ & 4.10 & 1.03 & 1.01 & 88 & 0 & 52.5 & 54.1 \\
\hline 17.5 & $12 / 5 / 0618: 00$ & 4.20 & 1.03 & 1.01 & 87 & 0 & 52.3 & 53.7 \\
\hline 18.0 & 12/5/06 18:30 & 4.32 & 1.04 & 1.01 & 89 & 0 & 52.2 & 53.7 \\
\hline 18.5 & 12/5/06 19:00 & 4.49 & 1.04 & 1.01 & 89 & 80 & 52.0 & 53.8 \\
\hline 19.0 & 12/5/06 19:30 & 3.66 & 1.04 & 1.01 & 90 & 0 & 52.0 & 53.6 \\
\hline 19.5 & $12 / 5 / 0620: 00$ & 4.93 & 1.04 & 1.01 & 88 & 0 & 53.6 & 54.5 \\
\hline 20.0 & $12 / 5 / 0620: 30$ & 5.15 & 1.04 & 1.01 & 91 & 0 & 52.7 & 54.2 \\
\hline 20.5 & $12 / 5 / 0621: 00$ & 3.44 & 1.04 & 1.01 & 90 & 0 & 52.8 & 54.3 \\
\hline 21.0 & $12 / 5 / 06$ 21:30 & 4.23 & 1.04 & 1.01 & 89 & 0 & 52.2 & 53.9 \\
\hline 21.5 & $12 / 5 / 0622: 00$ & 4.22 & 1.04 & 1.01 & 88 & 0 & 52.2 & 53.9 \\
\hline 22.0 & $12 / 5 / 06$ 22:30 & 4.21 & 1.03 & 1.01 & 89 & 0 & 52.2 & 54.3 \\
\hline 22.5 & 12/5/06 23:00 & 3.83 & 1.03 & 1.01 & 88 & 0 & 52.3 & 54.1 \\
\hline 23.0 & $12 / 5 / 0623: 30$ & 0.02 & 1.03 & 1.01 & 91 & 0 & 53.3 & 55.3 \\
\hline 23.5 & $12 / 6 / 060: 00$ & 3.48 & 1.03 & 1.01 & 92 & 0 & 53.6 & 54.9 \\
\hline 24.0 & $12 / 6 / 06$ 0:30 & 4.05 & 1.03 & 1.01 & 90 & 0 & 53.0 & 54.7 \\
\hline 24.5 & $12 / 6 / 061: 00$ & 4.20 & 1.03 & 1.01 & 89 & 0 & 53.0 & 54.5 \\
\hline 25.0 & $12 / 6 / 061: 30$ & 3.80 & 1.03 & 1.01 & 87 & 0 & 52.8 & 54.4 \\
\hline 25.5 & $12 / 6 / 062: 00$ & 4.20 & 1.03 & 1.01 & 86 & 0 & 52.7 & 54.3 \\
\hline 26.0 & $12 / 6 / 062: 30$ & 0.02 & 1.03 & 1.01 & 89 & 0 & 53.5 & 55.5 \\
\hline 26.5 & 12/6/06 3:00 & 4.10 & 1.03 & 1.01 & 92 & 0 & 53.9 & 55.3 \\
\hline 27.0 & 12/6/06 3:30 & 3.70 & 1.03 & 1.01 & 91 & 0 & 53.5 & 55.2 \\
\hline 27.5 & $12 / 6 / 064: 00$ & 4.00 & 1.03 & 1.01 & 88 & 0 & 53.2 & 54.8 \\
\hline 28.0 & $12 / 6 / 064: 30$ & 0 & 1.03 & 1.01 & 90 & 0 & 53.7 & 55.9 \\
\hline 28.5 & $12 / 6 / 065: 00$ & 4.09 & 1.03 & 1.01 & 91 & 0 & 53.9 & 55.4 \\
\hline 29.0 & $12 / 6 / 065: 30$ & 3.50 & 1.03 & 1.01 & 89 & 0 & 53.7 & 55.0 \\
\hline 29.5 & $12 / 6 / 066: 00$ & 0.02 & 1.03 & 1.01 & 91 & 0 & 53.8 & 56.2 \\
\hline 30.0 & $12 / 6 / 06$ 6:30 & 4.10 & 1.03 & 1.01 & 90 & 0 & 54.1 & 55.3 \\
\hline 30.5 & 12/6/06 7:00 & 4.20 & 1.03 & 1.01 & 88 & 0 & 53.7 & 55.0 \\
\hline 31.0 & $12 / 6 / 06$ 7:30 & 4.00 & 1.03 & 1.01 & 87 & 0 & 53.4 & 55.1 \\
\hline 31.5 & $12 / 6 / 068: 00$ & 4.90 & 1.03 & 1.01 & 92 & 0 & 53.2 & 55.2 \\
\hline 32.0 & $12 / 6 / 068: 30$ & 3.80 & 1.03 & 1.01 & 90 & 0 & 53.2 & 54.9 \\
\hline 32.5 & $12 / 6 / 06$ 9:00 & 3.70 & 1.03 & 1.01 & 88 & 0 & 53.0 & 54.7 \\
\hline 33.0 & 12/6/06 9:30 & 4.80 & 1.03 & 1.01 & 89 & 0 & 53.0 & 54.7 \\
\hline 33.5 & $12 / 6 / 0610: 00$ & 3.80 & 1.03 & 1.01 & 90 & 0 & 52.8 & 54.4 \\
\hline 34.0 & $12 / 6 / 0610: 30$ & 4.10 & 1.03 & 1.01 & 90 & 0 & 52.4 & 54.4 \\
\hline 34.5 & 12/6/06 11:00 & 4.00 & 1.03 & 1.01 & 90 & 0 & 52.5 & 54.1 \\
\hline 35.0 & $12 / 6 / 0611: 30$ & 3.80 & 1.03 & 1.01 & 90 & 0 & 52.3 & 54.1 \\
\hline 35.5 & $12 / 6 / 0612: 00$ & 3.90 & 1.03 & 1.01 & 90 & 0 & 52.1 & 53.9 \\
\hline
\end{tabular}

Page 31 of 37 
WSRC-STI-2007-00286, REVISON 0

Batch 4 Heating

\begin{tabular}{|c|c|c|c|c|c|c|c|c|}
\hline $\begin{array}{l}\text { Batch \#4 } \\
\text { Duration }\end{array}$ & Date/Time & $\begin{array}{l}\text { EVAP POT } \\
\text { PRESS \#1 } \\
\text { Ch1 BLUE }\end{array}$ & $\begin{array}{c}\text { SPG \#1 } \\
\text { Ch1 BLUE }\end{array}$ & $\begin{array}{c}\text { SPG \#2 } \\
\text { Ch2 RED }\end{array}$ & $\begin{array}{c}\text { EVAP POT } \\
\text { TEMP \#1 } \\
\text { Ch1 BLUE }\end{array}$ & $\begin{array}{l}\text { TUBE BDL } \\
\text { FL \#2 Ch2 } \\
\text { RED }\end{array}$ & $\begin{array}{l}\text { 2H TRUE } \\
\text { LVL \#1 Ch1 } \\
\text { BLUE }\end{array}$ & $\begin{array}{c}\text { 2H TRUE } \\
\text { LVL \#2 } \\
\text { Ch2 RED }\end{array}$ \\
\hline 0.5 & $12 / 7 / 0623: 30$ & 2.20 & 1.05 & 1.04 & 18 & 100 & 62.2 & 63.7 \\
\hline 1.0 & $12 / 8 / 060: 00$ & 2.10 & 1.05 & 1.04 & 26 & 92 & 62.4 & 64.0 \\
\hline 1.5 & $12 / 8 / 060: 30$ & 2.01 & 1.04 & 1.03 & 34 & 189 & 62.9 & 64.3 \\
\hline 2.0 & 12/8/06 1:00 & 2.16 & 1.04 & 1.03 & 46 & 194 & 63.2 & 64.7 \\
\hline 2.5 & $12 / 8 / 061: 30$ & 2.27 & 1.04 & 1.01 & 58 & 190 & 63.5 & 65.3 \\
\hline 3.0 & $12 / 8 / 062: 00$ & 2.40 & 1.02 & 1.01 & 71 & 300 & 64.2 & 65.8 \\
\hline 3.5 & $12 / 8 / 062: 30$ & 2.90 & 1.02 & 1.00 & 82 & 297 & 64.5 & 66.2 \\
\hline 4.0 & $12 / 8 / 063: 00$ & 2.80 & 1.02 & 1.00 & 92 & 0 & 65.0 & 66.5 \\
\hline 4.5 & $12 / 8 / 063: 30$ & 2.30 & 1.02 & 1.00 & 91 & 0 & 64.5 & 66.2 \\
\hline 5.0 & $12 / 8 / 064: 00$ & 2.70 & 1.02 & 1.00 & 90 & 30 & 64.1 & 66.1 \\
\hline 5.5 & $12 / 8 / 064: 30$ & 2.80 & 1.02 & 1.00 & 91 & 0 & 63.9 & 65.9 \\
\hline 6.0 & $12 / 8 / 065: 00$ & 2.70 & 1.02 & 1.00 & 89 & 0 & 64.1 & 65.7 \\
\hline 6.5 & $12 / 8 / 065: 30$ & 2.70 & 1.02 & 1.00 & 85 & 0 & 63.9 & 65.8 \\
\hline 7.0 & $12 / 8 / 066: 00$ & 3.00 & 1.02 & 1.00 & 92 & 0 & 63.9 & 66.4 \\
\hline 7.5 & $12 / 8 / 066: 30$ & 2.60 & 1.02 & 1.00 & 90 & 0 & 63.7 & 65.7 \\
\hline 8.0 & $12 / 8 / 067: 00$ & 3.10 & 1.02 & 1.00 & 88 & 0 & 63.4 & 65.2 \\
\hline 8.5 & $12 / 8 / 067: 30$ & 3.50 & 1.02 & 0.99 & 92 & 45 & 63.5 & 65.5 \\
\hline 9.0 & $12 / 8 / 068: 00$ & 2.90 & 1.02 & 1.00 & 94 & 0 & 63.6 & 65.5 \\
\hline 9.5 & $12 / 8 / 068: 30$ & 2.80 & 1.02 & 1.00 & 92 & 0 & 63.2 & 64.9 \\
\hline 10.0 & $12 / 8 / 069: 00$ & 3.00 & 1.02 & 1.00 & 90 & 0 & 62.7 & 64.7 \\
\hline 10.5 & $12 / 8 / 069: 30$ & 2.70 & 1.02 & 1.00 & 89 & 0 & 62.5 & 64.4 \\
\hline 11.0 & $12 / 8 / 0610: 00$ & 3.20 & 1.02 & 1.00 & 87 & 0 & 62.3 & 64.2 \\
\hline 11.5 & $12 / 8 / 0610: 30$ & 3.00 & 1.03 & 1.00 & 86 & 0 & 62.3 & 63.9 \\
\hline 12.0 & 12/8/06 11:00 & 2.60 & 1.02 & 1.00 & 86 & 0 & 62.2 & 63.9 \\
\hline 12.5 & 12/8/06 11:30 & 3.10 & 1.02 & 1.00 & 92 & 31 & 62.9 & 64.6 \\
\hline 13.0 & $12 / 8 / 0612: 00$ & 2.70 & 1.02 & 1.00 & 95 & 0 & 62.4 & 64.4 \\
\hline 13.5 & $12 / 8 / 0612: 30$ & 2.70 & 1.02 & 1.00 & 93 & 0 & 62.5 & 64.1 \\
\hline 14.0 & $12 / 8 / 0613: 00$ & 2.70 & 1.02 & 1.00 & 91 & 0 & 61.8 & 63.6 \\
\hline 14.5 & $12 / 8 / 0613: 30$ & 2.60 & 1.02 & 1.00 & 90 & 0 & 61.6 & 63.6 \\
\hline 15.0 & $12 / 8 / 0614: 00$ & 2.90 & 1.02 & 1.00 & 88 & 0 & 61.7 & 63.4 \\
\hline 15.5 & $12 / 8 / 0614: 30$ & 2.70 & 1.02 & 1.00 & 88 & 0 & 61.7 & 63.3 \\
\hline 16.0 & $12 / 8 / 0615: 00$ & 2.80 & 1.02 & 1.00 & 86 & 0 & 61.4 & 63.1 \\
\hline 16.5 & $12 / 8 / 0615: 30$ & 2.90 & 1.02 & 1.00 & 86 & 0 & 61.3 & 63.1 \\
\hline 17.0 & $12 / 8 / 0616: 00$ & 3.10 & 1.02 & 1.00 & 91 & 32 & 61.6 & 63.3 \\
\hline 17.5 & $12 / 8 / 0616: 30$ & 2.50 & 1.02 & 1.00 & 94 & 0 & 61.3 & 63.1 \\
\hline 18.0 & $12 / 8 / 0617: 00$ & 2.50 & 1.02 & 1.00 & 92 & 0 & 61.0 & 63.1 \\
\hline 18.5 & $12 / 8 / 0617: 30$ & 2.60 & 1.02 & 1.00 & 91 & 0 & 60.7 & 62.9 \\
\hline 19.0 & $12 / 8 / 0618: 00$ & 2.80 & 1.02 & 1.00 & 89 & 0 & 60.5 & 62.5 \\
\hline 19.5 & $12 / 8 / 0618: 30$ & 2.90 & 1.02 & 1.00 & 88 & 0 & 60.0 & 62.3 \\
\hline 20.0 & 12/8/06 19:00 & 3.10 & 1.00 & 1.00 & 87 & 0 & 60.0 & 62.0 \\
\hline 20.5 & $12 / 8 / 0619: 30$ & 3.00 & 1.00 & 1.00 & 92 & 100 & 60.0 & 62.0 \\
\hline 21.0 & $12 / 8 / 0620: 00$ & 2.80 & 1.00 & 1.00 & 92 & 0 & 60.0 & 62.0 \\
\hline 21.5 & $12 / 8 / 0620: 30$ & 2.70 & 1.00 & 1.00 & 90 & 0 & 60.0 & 61.0 \\
\hline 22.0 & $12 / 8 / 0621: 00$ & 2.60 & 1.00 & 1.00 & 89 & 0 & 60.0 & 61.0 \\
\hline 22.5 & $12 / 8 / 0621: 30$ & 2.90 & 1.00 & 1.00 & 87 & 0 & 60.0 & 61.0 \\
\hline 23.0 & $12 / 8 / 0622: 00$ & 3.00 & 1.00 & 1.00 & 86 & 0 & 59.0 & 61.0 \\
\hline 23.5 & $12 / 8 / 0622: 30$ & 2.40 & 1.00 & 1.00 & 95 & 0 & 60.0 & 62.0 \\
\hline 24.0 & $12 / 8 / 0623: 00$ & 2.20 & 1.00 & 1.00 & 94 & 0 & 59.0 & 61.0 \\
\hline 24.5 & $12 / 8 / 0623: 30$ & 2.00 & 1.00 & 1.00 & 92 & 0 & 59.0 & 61.0 \\
\hline 25.0 & 12/9/06 0:00 & 1.71 & 1.00 & 1.00 & 90 & 0 & 59.0 & 61.0 \\
\hline 25.5 & 12/9/06 0:30 & 1.90 & 1.00 & 1.00 & 89 & 0 & 59.0 & 61.0 \\
\hline 26.0 & 12/9/06 1:00 & 2.60 & 1.00 & 1.00 & 88 & 0 & 59.0 & 61.0 \\
\hline 26.5 & 12/9/06 1:30 & 0.00 & 1.00 & 1.00 & 95 & 0 & 60.0 & 61.0 \\
\hline 27.0 & $12 / 9 / 062: 00$ & 2.50 & 1.00 & 1.00 & 93 & 0 & 60.0 & 61.0 \\
\hline 27.5 & $12 / 9 / 062: 30$ & 2.40 & 1.00 & 1.00 & 91 & 0 & 60.0 & 61.0 \\
\hline 28.0 & $12 / 9 / 063: 00$ & 2.90 & 1.00 & 1.00 & 90 & 0 & 60.0 & 61.0 \\
\hline 28.5 & 12/9/06 3:30 & 2.00 & 1.00 & 1.00 & 89 & 0 & 59.0 & 60.0 \\
\hline 29.0 & $12 / 9 / 064: 00$ & 2.50 & 1.00 & 1.00 & 87 & 0 & 59.0 & 60.0 \\
\hline 29.5 & $12 / 9 / 064: 30$ & 2.60 & 1.00 & 1.00 & 86 & 0 & 59.0 & 60.0 \\
\hline 30.0 & $12 / 9 / 065: 00$ & 0.02 & 1.00 & 1.00 & 95 & 0 & 60.0 & 61.0 \\
\hline 30.5 & $12 / 9 / 065: 30$ & 1.70 & 1.00 & 1.00 & 95 & 0 & 60.0 & 61.0 \\
\hline 31.0 & $12 / 9 / 066: 00$ & 0.04 & 1.00 & 1.00 & 93 & 0 & 59.0 & 61.0 \\
\hline 31.5 & $12 / 9 / 066: 30$ & 2.50 & 1.00 & 1.00 & 93 & 0 & 59.0 & 60.0 \\
\hline 32.0 & $12 / 9 / 067: 00$ & 2.20 & 1.00 & 1.00 & 91 & 0 & 58.8 & 60.4 \\
\hline 32.5 & 12/9/06 7:30 & 2.40 & 1.02 & 1.00 & 89 & 0 & 58.8 & 60.7 \\
\hline 33.0 & $12 / 9 / 068: 00$ & 2.30 & 1.02 & 1.00 & 88 & 0 & 58.6 & 61.1 \\
\hline 33.5 & $12 / 9 / 068: 30$ & 2.60 & 1.03 & 1.00 & 89 & 0 & 58.7 & 60.5 \\
\hline 34.0 & 12/9/06 9:00 & 1.30 & 1.03 & 1.00 & 88 & 0 & 58.6 & 60.3 \\
\hline 34.5 & 12/9/06 9:30 & 2.20 & 1.03 & 1.01 & 87 & 0 & 58.4 & 60.1 \\
\hline 35.0 & 12/9/06 10:00 & 3.40 & 1.02 & 1.00 & 88 & 0 & 58.8 & 60.4 \\
\hline 35.5 & $12 / 9 / 0610: 30$ & 2.70 & 1.03 & 1.00 & 87 & 0 & 58.7 & 60.2 \\
\hline 36.0 & 12/9/06 11:00 & 2.60 & 1.03 & 1.00 & 86 & 0 & 58.4 & 60.1 \\
\hline
\end{tabular}

Cooling Batch 1

Page 32 of 37 


$\begin{array}{rc}\text { Cooling } & \\ \text { Batch \#1 } & \text { Date/Time } \\ 0.0 & 11 / 23 / 0719: 00 \\ 1.0 & 11 / 23 / 0720: 00 \\ 2.0 & 11 / 23 / 0721: 00 \\ 3.0 & 11 / 23 / 0722: 00 \\ 4.0 & 11 / 23 / 0723: 00 \\ 5.0 & 11 / 24 / 070: 00 \\ 6.0 & 11 / 24 / 071: 00 \\ 7.0 & 11 / 24 / 072: 00 \\ 8.0 & 11 / 24 / 073: 00 \\ 9.0 & 11 / 24 / 074: 00 \\ 10.0 & 11 / 24 / 075: 00 \\ 11.0 & 11 / 24 / 076: 00\end{array}$

Cooling Batch 2

$\begin{array}{rr}\text { Cooling } & \\ \text { Batch \#2 } & \text { Date/Time } \\ 0.0 & 12 / 1 / 075: 00 \\ 1.0 & 12 / 1 / 07 \text { 6:00 } \\ 2.0 & 12 / 1 / 077: 00 \\ 3.0 & 12 / 1 / 078: 00 \\ 4.0 & 12 / 1 / 07 \text { 9:00 } \\ 5.0 & 12 / 1 / 0710: 00 \\ 6.0 & 12 / 1 / 0711: 00 \\ 7.0 & 12 / 1 / 0712: 00 \\ 8.0 & 12 / 1 / 0713: 00 \\ 9.0 & 12 / 1 / 0714: 00 \\ 10.0 & 12 / 1 / 0715: 00 \\ 11.0 & 12 / 1 / 0716: 00 \\ 12.0 & 12 / 1 / 0717: 00 \\ 13.0 & 12 / 1 / 0718: 00 \\ 14.0 & 12 / 1 / 0719: 00 \\ 15.0 & 12 / 1 / 0720: 00 \\ 16.0 & 12 / 1 / 0721: 00 \\ 17.0 & 12 / 1 / 0722: 00 \\ 18.0 & 12 / 1 / 0723: 00 \\ 19.0 & 12 / 2 / 070: 00 \\ 20.0 & 12 / 2 / 071: 00\end{array}$

\begin{tabular}{|c|c|c|}
\hline EVAP POT & SPG \#1 & \\
\hline PRESS \#1 & Ch1 & SPG \#2 \\
\hline Ch1 BLUE & BLUE & Ch2 RED \\
\hline 2.80 & 1.05 & 1.02 \\
\hline 2.60 & 1.05 & 1.02 \\
\hline 3.20 & 1.05 & 1.03 \\
\hline 2.90 & 1.05 & 1.03 \\
\hline 2.90 & 1.05 & 1.03 \\
\hline 3.27 & 1.05 & 1.03 \\
\hline 2.90 & 1.05 & 1.03 \\
\hline 3.20 & 1.05 & 1.04 \\
\hline 3.06 & 1.05 & 1.04 \\
\hline 3.09 & 1.06 & 1.04 \\
\hline 3.09 & 1.06 & 1.04 \\
\hline 2.92 & 1.06 & 1.05 \\
\hline 3.13 & 1.07 & 1.05 \\
\hline 2.73 & 1.07 & 1.05 \\
\hline 2.90 & 1.07 & 1.06 \\
\hline 2.80 & 1.08 & 1.06 \\
\hline 2.80 & 1.08 & 1.06 \\
\hline 3.30 & 1.08 & 1.06 \\
\hline 0.04 & 1.08 & 1.06 \\
\hline 0.04 & 1.08 & 1.07 \\
\hline 0.05 & 1.08 & 1.07 \\
\hline
\end{tabular}

EVAP POT PRESS \#1 Ch1 BLUE

$$
2.10
$$$$
2.10
$$$$
1.97
$$$$
2.10
$$$$
2.00
$$$$
2.00
$$$$
2.11
$$$$
2.11
$$$$
22.00
$$$$
13.00
$$$$
0.04
$$

0.05

SPG \#1

Ch1 SPG \#2

BLUE Ch2 RED

$1.10 \quad 1.08$

1.10

1.11

1.12

1.13

1.12

1.10

1.10

1.10

1.10

1.10

$\begin{array}{crrr}\begin{array}{c}\text { EVAP POT } \\ \text { TEMP \#1 }\end{array} & \begin{array}{r}\text { EVAP T-B } \\ \text { PRESS \#1 }\end{array} & \begin{array}{r}\text { 2H TRUE } \\ \text { LVI Ch1 }\end{array} & \begin{array}{c}\text { 2H TRUE } \\ \text { LVL \#2 }\end{array} \\ \begin{array}{c}\text { Ch1 BLUE } \\ \text { Ch1 BLUE }\end{array} & \begin{array}{r}\text { BLUE } \\ \text { Ch2 RED }\end{array} \\ 82 & 0 & 46.0 & 47.0 \\ 82 & 28 & 47.0 & 47.0 \\ 76 & 22 & 45.6 & 46.9 \\ 63 & 21 & 45.1 & 46.4 \\ 52 & 26 & 44.8 & 46.0 \\ 46 & 25 & 44.7 & 46.0 \\ 39 & 26 & 44.4 & 45.6 \\ 37 & 26 & 44.3 & 45.4 \\ 30 & 24 & 44.7 & 45.9 \\ 30 & 14 & 45.0 & 46.0 \\ 30 & 19 & 43.0 & 45.3 \\ 30 & 19 & 44.0 & 45.0\end{array}$

$\begin{array}{crrr}\begin{array}{c}\text { EVAP POT } \\ \text { TEMP \#1 }\end{array} & \begin{array}{r}\text { EVAP T-B } \\ \text { PRESS \#1 }\end{array} & \begin{array}{c}\text { 2H TRUE } \\ \text { LVL \#1 }\end{array} & \begin{array}{c}\text { 2H TRUE } \\ \text { LVL \#2 }\end{array} \\ \text { Ch1 BLUE } & \text { Ch1 BLUE } & \text { Ch1 BLUE } & \text { Ch2 RED } \\ 90 & 28 & 54.0 & 55.9 \\ 86 & 27 & 53.4 & 55.4 \\ 82 & 28 & 53.1 & 54.7 \\ 81 & 28 & 53.1 & 54.6 \\ 80 & 27 & 52.9 & 54.6 \\ 79 & 27 & 52.7 & 54.2 \\ 76 & 27 & 52.6 & 54.0 \\ 75 & 28 & 52.5 & 53.8 \\ 74 & 27 & 52.3 & 53.7 \\ 73 & 28 & 52.1 & 53.4 \\ 72 & 28 & 52.1 & 53.4 \\ 69 & 35 & 51.9 & 53.1 \\ 61 & 35 & 51.5 & 52.8 \\ 53 & 34 & 51.3 & 52.5 \\ 45 & 33 & 51.0 & 52.2 \\ 39 & 35 & 51.0 & 52.0 \\ 36 & 34 & 50.7 & 52.0 \\ 33 & 34 & 50.6 & 52.0 \\ 31 & 34 & 50.4 & 51.8 \\ 30 & 34 & 50.4 & 51.8 \\ 29 & 35 & 50.4 & 51.8\end{array}$


Cooling Batch 3

\begin{tabular}{|c|c|c|c|c|c|c|c|c|}
\hline $\begin{array}{l}\text { Cooling } \\
\text { Batch \#3 }\end{array}$ & Date/Time & $\begin{array}{l}\text { EVAP POT } \\
\text { PRESS \#1 } \\
\text { Ch1 BLUE }\end{array}$ & $\begin{array}{c}\text { SPG \#1 } \\
\text { Ch1 BLUE }\end{array}$ & $\begin{array}{c}\text { SPG \#2 } \\
\text { Ch2 RED }\end{array}$ & $\begin{array}{c}\text { EVAP POT } \\
\text { TEMP \#1 } \\
\text { Ch1 BLUE }\end{array}$ & $\begin{array}{l}\text { EVAP T-B } \\
\text { PRESS \#1 } \\
\text { Ch1 BLUE }\end{array}$ & $\begin{array}{c}\text { 2H TRUE } \\
\text { LVL \#1 } \\
\text { Ch1 BLUE }\end{array}$ & $\begin{array}{c}\text { 2H TRUE } \\
\text { LVL \#2 } \\
\text { Ch2 RED }\end{array}$ \\
\hline 0.0 & 12/6/07 13:00 & 4.20 & 1.03 & 1.01 & 88 & 0 & 51.7 & 53.4 \\
\hline 1.0 & 12/6/07 14:00 & 4.30 & 1.04 & 1.01 & 83 & 96 & 51.6 & 53. \\
\hline 2.0 & 12/6/07 15:00 & 3.90 & 1.04 & 1.02 & 73 & 33 & 51.0 & 52. \\
\hline 3.0 & 12/6/07 16:00 & 3.90 & 1.04 & 1.02 & 62 & 38 & 50.5 & 51 \\
\hline 4.0 & 12/6/07 17:00 & 4.00 & 1.04 & 1.03 & 54 & 34 & 50.3 & 51 \\
\hline 5.0 & 12/6/07 18:00 & 4.30 & 1.06 & 1.04 & 46 & 38 & 50.8 & 51. \\
\hline 6.0 & 12/6/07 19:00 & 4.39 & 1.06 & 1.04 & 42 & 32 & 49.8 & 51 \\
\hline 7.0 & 12/6/07 20:00 & 4.64 & 1.06 & 1.05 & 37 & 28 & 49.7 & \\
\hline
\end{tabular}

Cooling Batch 4

$\begin{array}{rc}\begin{array}{c}\text { Cooling } \\ \text { Batch \#4 }\end{array} & \begin{array}{c}\text { Date/Time } \\ 0.0\end{array} \\ 12 / 23 / 07 ~ 19: 00 \\ 1.0 & 12 / 23 / 0720: 00 \\ 2.0 & 12 / 23 / 0721: 00 \\ 3.0 & 12 / 23 / 0722: 00 \\ 4.0 & 12 / 23 / 0723: 00 \\ 5.0 & 12 / 24 / 07 \text { 0:00 } \\ 6.0 & 12 / 24 / 071: 00 \\ 7.0 & 12 / 24 / 072: 00 \\ 8.0 & 12 / 24 / 073: 00 \\ 9.0 & 12 / 24 / 074: 00 \\ 10.0 & 12 / 24 / 075: 00 \\ 11.0 & 12 / 24 / 076: 00\end{array}$

\begin{tabular}{rrr}
$\begin{array}{rrr}\text { EVAP POT } \\
\text { PRESS \#1 }\end{array}$ & \multicolumn{1}{c}{ SPG \#1 } & \multicolumn{1}{c}{ SPG \#2 } \\
Ch1 BLUE & Ch1 BLUE & Ch2 RED \\
2.10 & 1.10 & 1.08 \\
2.10 & 1.10 & 1.08 \\
1.97 & 1.11 & 1.09 \\
2.10 & 1.12 & 1.10 \\
2.00 & 1.13 & 1.10 \\
2.00 & 1.13 & 1.11 \\
2.11 & 1.13 & 1.11 \\
2.11 & 1.13 & 1.11 \\
22.00 & 1.12 & 1.11 \\
13.00 & 1.10 & 1.10 \\
0.04 & 1.10 & 1.10 \\
0.05 & 1.10 & 1.10
\end{tabular}

\begin{tabular}{|c|c|c|c|}
\hline $\begin{array}{l}\text { EVAP POT } \\
\text { TEMP \#1 }\end{array}$ & $\begin{array}{l}\text { EVAP T-B } \\
\text { PRESS \#1 }\end{array}$ & $\begin{array}{c}\text { 2H TRUE } \\
\text { LVL \#1 Ch1 }\end{array}$ & $\begin{array}{c}\text { 2H TRUE } \\
\text { LVL \#2 }\end{array}$ \\
\hline Ch1 BLUE & Ch1 BLUE & BLUE & Ch2 RED \\
\hline 82 & 0 & 46.0 & 47.0 \\
\hline 82 & 28 & 47.0 & 47.0 \\
\hline 76 & 22 & 45.6 & 46.9 \\
\hline 63 & 21 & 45.1 & 46.4 \\
\hline 52 & 26 & 44.8 & 46.0 \\
\hline 46 & 25 & 44.7 & 46.0 \\
\hline 39 & 26 & 44.4 & 45.6 \\
\hline 37 & 26 & 44.3 & 45.4 \\
\hline 30 & 24 & 44.7 & 45.9 \\
\hline 30 & 14 & 45.0 & 46.0 \\
\hline 30 & 19 & 43.0 & 45.3 \\
\hline 30 & 19 & 44.0 & 45.0 \\
\hline
\end{tabular}


Neutralization Batch 1

\begin{tabular}{|c|c|}
\hline $\begin{array}{c}\text { Neutralization } \\
\text { Batch \#1 }\end{array}$ & Date/Time \\
\hline 0.0 & 11/28/06 0:00 \\
\hline 1.0 & 11/28/06 1:00 \\
\hline 2.0 & 11/28/06 2:00 \\
\hline 3.0 & 11/28/06 3:00 \\
\hline 4.0 & 11/28/06 4:00 \\
\hline 5.0 & 11/28/06 5:00 \\
\hline 6.0 & 11/28/06 6:00 \\
\hline 7.0 & 11/28/06 7:00 \\
\hline 8.0 & 11/28/06 8:00 \\
\hline 9.0 & 11/28/06 9:00 \\
\hline 10.0 & 11/28/06 10:00 \\
\hline 11.0 & 11/28/06 11:00 \\
\hline 12.0 & 11/28/06 12:00 \\
\hline 13.0 & 11/28/06 13:00 \\
\hline 14.0 & 11/28/06 14:00 \\
\hline 15.0 & 11/28/06 15:00 \\
\hline
\end{tabular}

Neutralization Batch 2

$\begin{array}{rc}\begin{array}{c}\text { Neutralization } \\ \text { Batch \#2 }\end{array} & \begin{array}{c}\text { Date/Time } \\ 0.0\end{array} \\ 12 / 1 / 06 & 0: 00 \\ 1.0 & 12 / 1 / 061: 00 \\ 2.0 & 12 / 1 / 06 ~ 2: 00 \\ 3.0 & 12 / 1 / 063: 00 \\ 4.0 & 12 / 1 / 064: 00 \\ 5.0 & 12 / 1 / 065: 00 \\ 6.0 & 12 / 1 / 066: 00 \\ 7.0 & 12 / 1 / 067: 00 \\ 8.0 & 12 / 1 / 068: 00 \\ 9.0 & 12 / 1 / 069: 00 \\ 10.0 & 12 / 1 / 0610: 00 \\ 11.0 & 12 / 1 / 0611: 00 \\ 12.0 & 12 / 1 / 0612: 00 \\ 13.0 & 12 / 1 / 0613: 00 \\ 14.0 & 12 / 1 / 0614: 00 \\ 15.0 & 12 / 1 / 0615: 00\end{array}$

\begin{tabular}{|c|c|c|c|c|c|c|}
\hline $\begin{array}{l}\text { EVAP POT } \\
\text { PRESS \#1 }\end{array}$ & $\begin{array}{c}\text { SPG \#1 } \\
\text { Ch1 }\end{array}$ & $\begin{array}{l}\text { SPG \#2 } \\
\text { Ch? RFD }\end{array}$ & $\begin{array}{c}\text { EVAP POT } \\
\text { TEMP \#1 } \\
\text { Ch1 BLUE }\end{array}$ & $\begin{array}{l}\text { EVAP T-B } \\
\text { PRESS \#1 } \\
\text { Ch1 BLUE }\end{array}$ & $\begin{array}{c}\text { 2H TRUE } \\
\text { LVL \#1 } \\
\text { Ch1 BLUE }\end{array}$ & $\begin{array}{c}\text { 2H TRUE } \\
\text { LVL \#2 } \\
\text { Ch2 RED }\end{array}$ \\
\hline $\begin{array}{r}\text { Ch1 BLUE } \\
13.6\end{array}$ & $\begin{array}{l}\text { BLUE } \\
1.13\end{array}$ & $\begin{array}{r}\text { Ch2 RED } \\
1.11\end{array}$ & $\begin{array}{r}\text { Ch1 BLUE } \\
26\end{array}$ & $\begin{array}{r}\text { Ch1 BLUE } \\
27\end{array}$ & $\begin{array}{r}\text { Ch1 BLUE } \\
44.4\end{array}$ & $\begin{array}{r}\text { Cn2 RED } \\
45.5\end{array}$ \\
\hline 13.6 & 1.13 & 1.11 & 24 & 27 & 44.4 & 45.4 \\
\hline 14.0 & 1.13 & 1.11 & 24 & 27 & 44.3 & 45.3 \\
\hline 14.0 & 1.13 & 1.11 & 23 & 72 & 44.3 & 45.4 \\
\hline 14.0 & 1.13 & 1.11 & 23 & 72 & 44.4 & 45.4 \\
\hline 13.0 & 1.13 & 1.11 & 39 & 72 & 53.8 & 55.3 \\
\hline 13.0 & 1.13 & 1.10 & 35 & 73 & 53.8 & 55.2 \\
\hline 12.8 & 1.10 & 1.11 & 32 & 73 & 53.0 & 54.0 \\
\hline 12.0 & 1.10 & 1.11 & 30 & 73 & 53.0 & 54.0 \\
\hline 12.6 & 1.20 & 1.10 & 37 & 73 & 59.0 & 60.0 \\
\hline 12.2 & 1.20 & 1.10 & 37 & 30 & 59.0 & 61.0 \\
\hline 12.2 & 1.20 & 1.10 & 36 & 30 & 60.0 & 61.0 \\
\hline 12.3 & 1.20 & 1.10 & 34 & 31 & 60.0 & 61.0 \\
\hline 12.8 & 1.20 & 1.10 & 34 & 30 & 60.6 & 62.0 \\
\hline 12.2 & 1.20 & 1.10 & 33 & 30 & 60.6 & 62.0 \\
\hline 12.3 & 1.20 & 1.10 & 33 & 30 & 60.6 & 62.0 \\
\hline
\end{tabular}

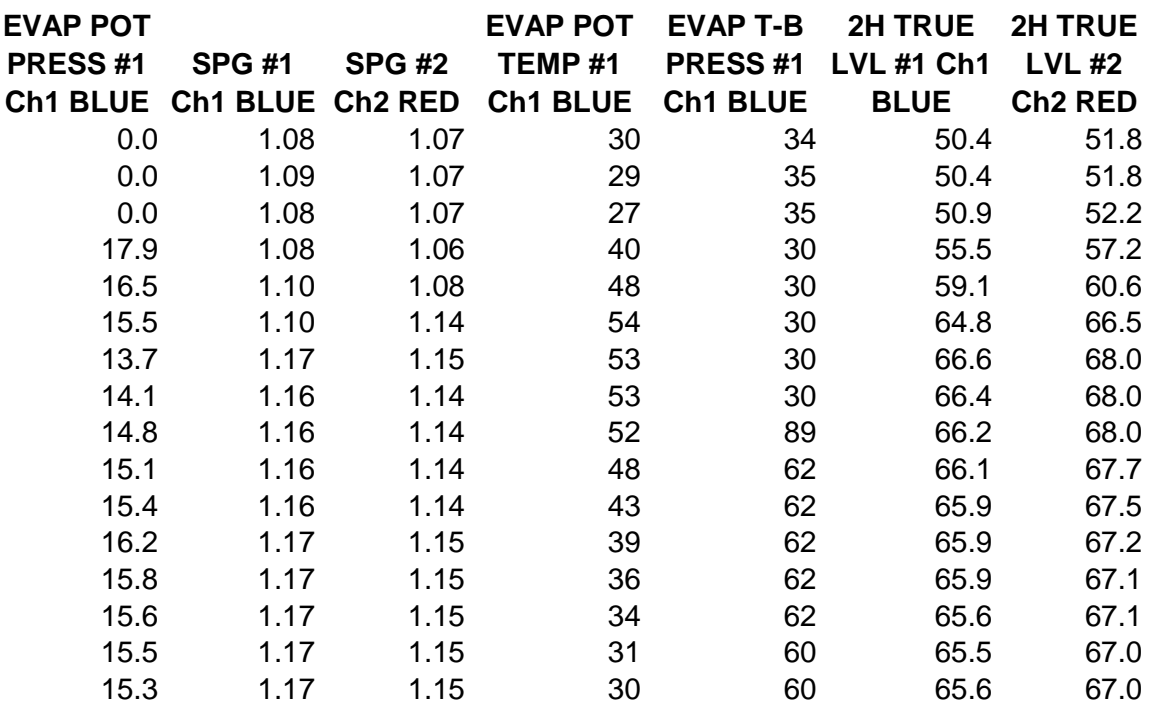


Neutralization Batch 3

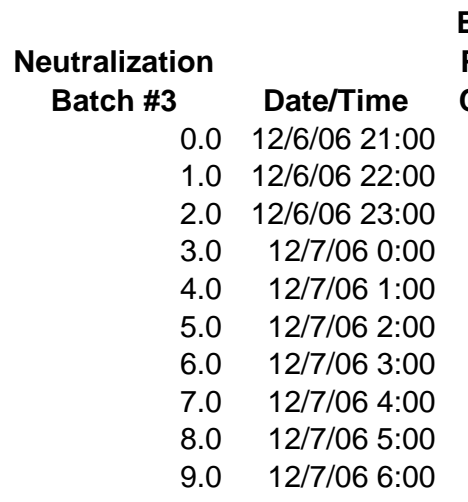

PRESS

SPG \#1 SPG \#2

$19.9 \quad 1.06$

$19.1 \quad 1.07$

18.6

18.9

19.8

0.0

19.8

20.0

19.8

20.0

Neutralization Batch 4

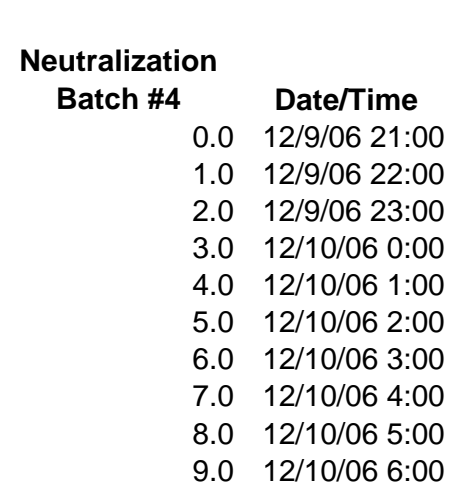

\begin{tabular}{rrr}
$\begin{array}{r}\text { EVAP POT } \\
\text { PRESS \#1 }\end{array}$ & \multicolumn{1}{c}{ Ch1 } & SPG \#2 \\
Ch1 BLUE & BLUE & Ch2 RED \\
0.1 & 1.00 & 1.00 \\
16.3 & 1.00 & 1.00 \\
16.0 & 1.00 & 1.00 \\
15.7 & 1.00 & 1.00 \\
15.1 & 1.10 & 1.00 \\
15.1 & 1.10 & 1.00 \\
15.0 & 1.10 & 1.10 \\
15.0 & 1.10 & 1.10 \\
15.0 & 1.10 & 1.10 \\
15.0 & 1.10 & 1.10
\end{tabular}

\begin{tabular}{|rrrr}
$\begin{array}{c}\text { EVAP POT } \\
\text { TEMP \#1 }\end{array}$ & $\begin{array}{r}\text { EVAP T-B } \\
\text { PRESS \#1 }\end{array}$ & $\begin{array}{r}\text { 2H TRUE } \\
\text { LVL \#1 }\end{array}$ & $\begin{array}{r}\text { 2H TRUE } \\
\text { LVL \#2 }\end{array}$ \\
$\begin{array}{rrrr}\text { Ch1 BLUE } \\
\text { Ch1 BLUE }\end{array}$ & $\begin{array}{r}\text { Ch1 BLUE } \\
\text { Ch2 RED }\end{array}$ \\
\hline 34 & 32 & 49.9 & 51.0 \\
\hline 31 & 28 & 49.9 & 52.2 \\
\hline 51 & 28 & 57.8 & 52.2 \\
\hline 44 & 32 & 57.6 & 59.8 \\
\hline 42 & 33 & 59.1 & 59.5 \\
42 & 32 & 63.0 & 61.3 \\
43 & 32 & 63.0 & 64.5 \\
43 & 32 & 63.0 & 64.6 \\
\hline 36 & 32 & 62.8 & 64.5 \\
43 & 32 & 62.8 & 64.7
\end{tabular}

$\begin{array}{rrrr}\begin{array}{c}\text { EVAP POT } \\ \text { TEMP \#1 }\end{array} & \begin{array}{r}\text { EVAP T-B } \\ \text { PRESS \#1 }\end{array} & \begin{array}{c}\text { 2H TRUE } \\ \text { LVL \#1 Ch1 }\end{array} & \begin{array}{c}\text { 2H TRUE } \\ \text { LVL \#2 }\end{array} \\ \begin{array}{rrrr}\text { Ch1 BLUE } \\ \text { Ch1 BLUE }\end{array} & \begin{array}{rlr}\text { BLUE } \\ \text { Ch2 RED }\end{array} \\ 30 & 27 & 56.0 & 57.0 \\ 29 & 34 & 57.0 & 58.0 \\ 49 & 32 & 64.0 & 65.8 \\ 44 & 32 & 65.0 & 67.0 \\ 45 & 32 & 69.0 & 71.0 \\ 41 & 32 & 71.0 & 72.0 \\ 37 & 32 & 71.0 & 72.0 \\ 34 & 32 & 70.0 & 72.0 \\ 32 & 32 & 70.0 & 72.0 \\ 30 & 32 & 70.0 & 72.0\end{array}$




\section{REFERENCES}

${ }^{1}$ W. R. Wilmarth, M. C. Thompson, C. J. Martino, V. H. Dukes, J. T. Mills, C. Boley, and B. L. Lewis, "Nitric Acid Cleaning of a Sodalite - Sodium Diuranate Scale in High Level Waste Evaporators," WSRC-MS-2001-00741, Rev. 0, October 4, 2001.

${ }^{2}$ W. R. Wilmarth, C. J. Coleman, J. C. Hart, and W. T. Boyce, "Characterization of Samples from the 242-16H Evaporator Wall," WSRC-TR-2000-00089, March 20, 2000

${ }^{3}$ W. R. Wilmarth, C. J. Coleman, A. R. Jurgensen, W. M. Smith, J. C. Hart, W. T. Boyce, D. Missmer, and C. M. Conley, "Characterization and Dissolution Studies of Samples from the 24216H Evaporator," WSRC-TR-2000-00038, Rev. 0, January 31, 2000.

${ }^{4}$ Barnes, M. C.; Addai-Mensah, J.; Gerson, A. R. J., Crystal Growth, 200 (1999), 251-264.

${ }^{5}$ Gasteiger, H. A.; Fredrick, W. J.; Streisel, R. C., J. Eng. Chem. Res., Vol 31, 1992, 1190.

${ }^{6}$ Buhl, J.; Löns, J., J. Alloys and Compounds, 235 (1996), 41.

${ }^{7}$ Kumada, N.; Wetrum, E. F.; Hemingway, B. S.; Zolotov, M. Y.; Semenov, Y. V.;

Khodakovsky, I. L.; Anovitz, L. M., J. Chem. Thermodynamics, 1995, 27, 1119.

${ }^{8}$ W. R. Wilmarth, M. C. Thompson, C. J. Martino, V. H. Dukes, J. T. Mills, C. Boley, and B. L. Lewis, "Nitric Acid Cleaning of a Sodalite - Sodium Diuranate Scale in High Level Waste Evaporators," WSRC-MS-2001-00741, Rev. 0, October 4, 2001.

${ }^{9}$ C. S. Boley, M. C. Thompson, W. R. Wilmarth, K. G. Brown, "Technical Basis for the 24216H Evaporator Cleaning Process (U)," WSRC-TR-2000-00211, Rev. 1, November 7, 2000.

${ }^{10}$ W. R. Wilmarth, "Technical Requirements for Dispositioning Tank 40H Decants, SRT-LWP2001-00032, Rev. 1, March 20, 2001.

11 W. R. Wilmarth, R. C. Sullivan and V. H. Dukes, "Nitric Acid Cleaning studies of Scale Samples from the $2 \mathrm{H}$ Evaporator Pot to Support Sodium Aluminosilicate Dissolution," WSRCSTI-2006-00249, August 10, 2006.

${ }^{12}$ K. Martin and M. A. Pettis, "1-Liter Uranium Bearing 2H Scale Cleaning Acid Simulant Neutralization," WSRC-TR-2006-00258, Rev. 0, July 31, 2006.

${ }^{13}$ D. J. Adamson, "Bench-Scale Evaporator Neutralization Testing," WSRC-TR-2006-00248, Rev. 0, July 2006

${ }^{14}$ D. J. Adamson, "In Pot Neutralization and Full-Scale Transfer Demonstration," SNRL-PSE2006-00177, August 15, 2006.

${ }^{15}$ M. D. Hopkins and D. D. Walker, "FLOWSHEET AND OPERATING PLAN FOR THE 24216H EVAPORATOR ACID CLEANING PROCESS USING NEUTRALIZATION IN THE EVAPORATOR POT (U)," WSRC-TR-2006-0259, Rev. 0, October 26, 2006.

${ }^{16}$ K. Kwon, "2H Evaporator Pot Liquid Cooling with Tube Bundle, Lance Air, Cell Ventilation, an Wall Heat Loss," HLW-STE-2000-00323, August 16, 2000.

${ }^{17}$ C. S. Boley, M. C. Thompson, W. R. Wilmarth, K. G. Brown, "Technical Basis for the 24216H Evaporator Cleaning Process (U)," WSRC-TR-2000-00211, Rev. 1, November 7, 2000

${ }^{18}$ W. R. Wilmarth, C. J. Martino, J. T. Mills, and V. H. Dukes, "Results of Chemical Cleaning the 242-16H (2H) Evaporator at the Savannah River Site," WSRC-TR-2001-00412, Rev. 0, August 31, 2001. 